\title{
Der Wille bei Descartes
}

\section{Descartes' Conception of the Will}

\author{
Mehmet Arslan ${ }^{1}$ (])
}

'Asst. Prof., Eskisehir Osmangazi University, Faculty of Economics and Administrative Sciences, Department of Business Administration, Department of Commercial Law, Eskisehir, Turkey

\section{ORCID: M.A. 0000-0002-3928-8887}

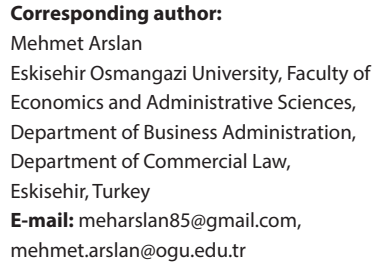

Citation: Arslan, Mehmet. (2020). “Der Wille bei Descartes" Felsefe Arkivi-Archives of Philosophy, 53: 55-89.

https://doi.org/10.26650/arcp.815512

\section{ZUSAMMENFASSUNG}

In den Anfängen seiner Philosophie äußerte sich Descartes über den epistemologischen Stellenwert des Willens nur allgemein. Nach Descartes hat Gott dem menschlichen Geist bereits die ersten Ideen gegeben, damit er die ebenfalls von Gott geschaffene physische Welt überhaupt wahrnehmen kann. Für ihn ist die dazu zuständige menschliche Fakultät unter anderem der Wille. Angesichts der immer wieder gemachten Erfahrung, dass die Menschen Irrtümer begehen, muss sich Descartes in der Fortsetzung seiner Philosophie mit der Frage auseinandersetzen, wie sich der Wille irrt und warum. Für die cartesianische Erklärung des ersten Teils der Frage ist entscheidend, wie der Geist im Zusammenwirken des Willens und des Verstandes den Erkenntnisakt vollzieht. Als Gründe der Irrtümer nennt Descartes die Grenzen des menschlichen Verstandes sowie die Unaufmerksamkeit der Menschen. Ob der Wille insbesondere bei der genannten Unaufmerksamkeit frei ist, sodass dessen Inhaber zur Verantwortung gezogen werden kann, ist eine weitere Frage, zu der Descartes Stellung beziehen muss. In den Meditationen entwickelt er eine Freiheitslehre, die jedoch auf Einwände seitens Hobbes stößt. Später in zwei Briefen an Mesland modifiziert er seine Freiheitslehre. Die Freiheit, die er hierbei annimmt, ist für Descartes hinreichend, um die Verantwortlichkeit des Menschen für sein Fehlverhalten anzunehmen, auch wenn Gott selbst den Menschen mit einem Willensvermögen erschaffen hat bzw. die Ausübung dieser Freiheit duldet. Stichwörter: Ursache des Irrtums, Cartesianische Freiheit, Gott und Freiheit des Willens, Verantwortlichkeit und Prädestination

\section{ABSTRACT}

In the early days of his philosophy, Descartes made only general statements about the epistemological value of the will. According to Descartes, God gave the human mind the first ideas so that it could perceive the physical world, which was also created by God. For Descartes, the human faculty in charge of this operation is, among other things, the will. In view of repeated experiences of human beings judging erroneously, Descartes must, in the continuation of his philosophy, deal with the questions of how and why the will makes judgement errors. For the Cartesian explanation of the first part of the question, it is crucial how the mind, in cooperation of the will and "understanding", carries out the act of cognition. Descartes names the limits of the human understanding and the inattention of people as reasons for judgement errors. Whether the will is free enough that its owner can be held 
responsible, especially considering the aforementioned inattention, is another question on which Descartes must take a position. In the Meditations he develops a conception of freedom, which, however, meets with some objections from Hobbes. Later in two letters to Mesland, he modifies his conception of freedom. For Descartes, the freedom he assumes is sufficient to justify man's responsibility for his wrongdoing, even if God himself created man with a will or tolerates the exercise of this freedom.

Keywords: Cause of Judgement Errors, Cartesian Freedom, God and Freedom of Will, Accountability and Predestination

\section{Einführung}

Es war das große Projekt des Naturwissenschaftlers Descartes, die Wurzeln seines Baums der Wissenschaften mit einer prima philosophia zu schlagen. ${ }^{1}$ Ihm ging es dabei um nicht weniger als um das Vorhaben, die damals herrschende aristotelische Wissenschaftstheorie mit einer neuen zu ersetzen. Dafür hatte Descartes allen Grund. Das Aufkommen eines mechanistischen Weltbildes, das zu seiner Zeit vor allem mit Galileo Galileis Entdeckungen und der Idee einer hinreichenden und intelligiblen Kausalität in der Natur seine Erfolge verbuchte, brachte nicht nur die aristotelische Lehre von beseelten Substanzen und der finalen Kausalität in Bedrängnis, ${ }^{2}$ sondern veranlasste auch eine Aufbruchstimmung in den Wissenschaften, deren Voranschreiten seit Jahrhunderten der aristotelische Syllogismus der mittelalterlichen kirchlichen Philosophie im Wege stand.

Vor diesem Hintergrund sah sich Descartes vor der Aufgabe gestellt, den neuen Wissenschaften eine erste Philosophie voranzustellen, die zwei Ziele erfüllen sollte: die Gewissheit der neuen wissenschaftlichen Erkenntnisse zu gewährleisten und die neue Wissenstheorie mit dem Glauben in Übereinstimmung zu bringen.

Im Hinblick auf die erste Fragestellung wehrt sich Descartes gegen die skeptische Philosophie mit einer Evidenzforderung, die nur Erkenntnisse mit der Gewissheit, dass sie intuitiv klar und distinkt sind, zulässt. Seiner Erkenntnistheorie legt er eine dualistische Metaphysik zugrunde, die ontologisch zwischen geistigen und körperlichen Substanzen unterscheidet. ${ }^{3}$ Das Fundament seiner Philosophie des Geistes macht seine wohl bekannteste Schlussfolgerung aus, nämlich „Ich denke, also bin ich“ („Je pense, donc je suis“ oder „ego cogito, ergo sum“). ${ }^{4}$ Nichts außer einem denkenden Ding ist Descartes und nichts mehr außer sich selbst als Geist weißt er mit der geforderten Evidenz, bis er durch reines Denken die Existenz eines theistischen Gottes bewiesen haben will. ${ }^{5}$ Erst dann ist es ihm möglich, Erkenntnisse über die Welt der zweiten Substanz, nämlich des Körpers zu erlangen. Der vollkommene, allmächtige und allgütige Gott, der wie alles andere auch ihn erschaffen und mit den Vermögen des Verstandes und des Willen ausgestattet

1 Hierzu siehe mehr Perler, Descartes, S. 39.

2 Für die Einwände Descartes gegen die aristotelische Seelenlehre siehe auch seinen Brief v. Mai 1641 an Regius (CSMK, 181 ff.; AT III, 371 ff.)

3 Hierzu siehe etwa seinen Brief v. Mai 1641 an Regius (CSMK, 182; AT III, 372)

4 AT VI, 32; hierzu siehe auch Perler, Descartes, S. 24.

5 AT VII, $49 \mathrm{ff}$. und 52. 
hat, ${ }^{6}$ sorgt dafür, dass der Mensch außer von sich selbst als ein denkendes Ding auch verlässliches und wahres Wissen von der körperlichen Naturen haben kann. ${ }^{7}$

Bevor Descartes auf der Grundlage einer dualistischen Metaphysik und unter den Annahmen, dass sein wahres Wesen im Denken besteht und ein theistischer Gott existiert, seine Erkenntnistheorie weiter ausbaut, hat er sich einer Problematik zu stellen: gleichgültig, wie verlässlich und sicher seine Erkenntnistheorie Wissen zu generieren vermag, erfahren die Menschen immer wieder, dass sie sich irren. ${ }^{8}$ Dabei hat er nicht Ursachen der Irrtümer zu erklären, die aus den Schwächen oder den Täuschungen der Sinnen, des Vorstellungsvermögens oder des Gedächtnisses herrühren. Descartes hat in diesem Stadium seiner Philosophie der Verlässlichkeit dieser Vermögen bereits eine Absage erteilt ${ }^{9}$ und lediglich das geistig-rationale Einsichtsvermögen zum Erkenntnisgewinnen für tauglich erklärt. ${ }^{10} \mathrm{Ihm}$ geht es also hierbei um die Irrtümer des Geistes, denen es eigen ist, dass sie auf rein mentaler Ebene, also bei der Tätigkeit des Geistes entstehen. ${ }^{11}$ In demselben Zusammenhang muss Descartes Wege vorweisen, die eine sichere bzw. irrtumsfreie Erkenntniserlangung des Geistes ermöglichen. Zwar ist der Gott, dessen Existenz er beweist, Garant der Wahrheiten in mehrerlei Hinsicht. ${ }^{12}$ Descartes muss allerdings auch Leser in Betracht ziehen, die dem genannten Beweis nicht beipflichten würden, die er aber von der Idee einer irrtumsfreien Erkenntnisgewinnung durch den Geist überzeugen möchte. ${ }^{13}$

Vorauszuschicken ist an dieser Stelle in aller Kürze Descartes' Begründung für den sich irrenden Geist: der Irrtum entsteht, wenn der Wille trotz des Fehlens einer klaren und distinkten Idee von einem Ding voreilig seine Zustimmung für deren Existenz erteilt. ${ }^{14}$ Da der Wille aber indifferent ist, kann er sich in diesen Fällen eines Urteils auch enthalten. Sobald er aber eine klare und distinkte Idee durch den Verstand vorgelegt bekommt, folgt er diesem spontan. Dieser Umstand vermindert nach Descartes keineswegs die Freiheit des Willens, die gerade mit den beiden beschriebenen Vermögen eine irrtumsfreie Erkenntniserlangung ermöglicht. ${ }^{15}$

Es ist einer der folgenreichsten Thesen Descartes, dass der Wille neben dem Verstand eine eigenständige Operationskraft in der Anwendung des Urteilsvermögens hat und dabei indifferent bzw. frei ist. Descartes' Äußerungen über den Willen sind nicht darauf begrenzt. Wie unten noch aufzuzeigen sein wird, hat er sich nicht nur an weiteren Stellen der Meditationen, ${ }^{16}$ sondern auch in früheren und späteren Schriften der Frage des Willens gewidmet. ${ }^{17}$

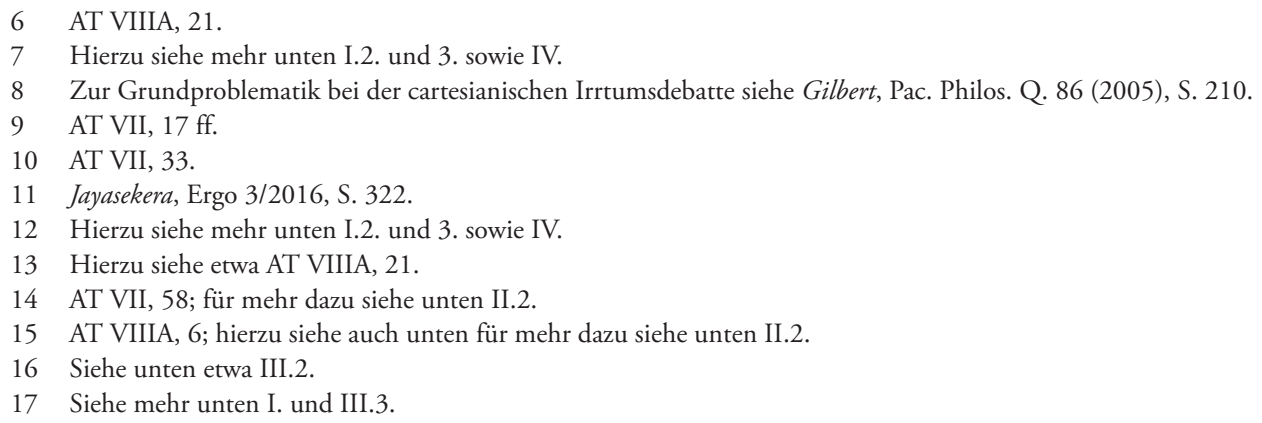


Wichtige Werke seiner einschlägigen Philosophie sind zunächst zwei Abhandlungen, die er schon in früheren Jahren seiner philosophischen Tätigkeit verfasst hat: aus dem Jahr 1619 Regulae ad directionem ingenii (Regeln zur Ausrichtung der Erkenntniskraft) und aus dem Jahr 1637 Discours de la méthode pour bien conduire sa raison et chercher la verité dans les sciences (Abhandlung über die Methode, richtig zu denken und die Wahrheit in den Wissenschaften zu suchen). 1641 schrieb Descartes dann sein berühmtes philosophisches Werk, nämlich Meditationes de prima philosophia, in qua Dei existentia et animae immortalitas demonstratur (Meditationen über die Erste Philosophie, in welcher die Existenz Gottes und die Unsterblichkeit der Seele bewiesen wird). ${ }^{18}$

Für die Zwecke dieses Beitrags hat Descartes eine umfassende Stellung zu dem Willen zuerst in den Meditationen bezogen. Dort ist die Rolle des Willens in Zusammenhang mit seinem gesamtphilosophischen Projekt aufgeführt. Dies bedeutet allerdings nicht, dass seine früheren sowie späteren Schriften, in denen er sich über den Willen geäußert hat, außer Acht zu lassen sind. Dies gilt insbesondere der Regulae, dem Discours de la méthode und auch seinem früheren und späteren Briefverkehr, zumal Descartes in letzteren einige Modifikationen vorgenommen hat. Vielmehr müssen diese mit den Meditationen in Beziehung gesetzt werden und zwar dergestalt, ob und inwiefern Descartes eine in sich geschlossene und überzeugende Willenslehre hatte bzw. wie er seine Philosophie diesbezüglich entwickelt hat oder auch modifiziert hat. $\mathrm{Zu}$ diesem Zweck werden im Folgenden einschlägigen Passagen seiner Werke chronologisch zum Verständnis der Willenslehre Descartes herangezogen.

In der Literatur lässt sich bei Descartes' Interpretationen bezüglich der Willenstheorie eine wichtige Differenzierung im Ansatz feststellen, die bei den anschließenden Erörterungen des Willens von Bedeutung bleibt. Während einige Autoren Descartes vor dem Hintergrund der in der Philosophie im Allgemeinen geführten Debatte um den Willen und dessen Freiheit einzuordnen versuchen, ${ }^{19}$ so sind andere darum bemüht, die Willenstheorie Descartes innerhalb seiner eigenen Metaphysik zu eruieren. ${ }^{20}$ Diese Differenzierung hat im Ansatz Auswirkungen auf die abschließenden Bewertungen. Interessiert sich der erste Ansatz für die Fragestellung, ob Descartes Willenstheorie deterministischer, indeterministischer oder kompatibilistischer Prämisse folgt, so hat der zweite Ansatz vergleichsweise keine scharfen Bewertungsrahmen und kann aus dem Umstand Vorteile erzielen, Descartes Willenstheorie auch außerhalb der Grenzen der allgemein geführten Debatte verorten zu können. Die Vorzugswürdigkeit des zweiten Ansatzes liegt nicht nur darin, Descartes Willenstheorie ohne Berücksichtigung einiger vorgefertigter Schablonen zu erfassen versuchen, sondern auch darin, Descartes Lehre gleichwohl nach den Grundgedanken der bereits genannten Positionen, nämlich des Determinismus, Indeterminismus oder Kompatibilismus prüfen zu können. Der vorliegende Beitrag schließt sich daher nicht zuletzt aus diesen Gründen dem zweiten Ansatz an.

18 Zur Entstehung siehe Perler, Descartes, S. 26.

19 Siehe etwa Embry, Descartes on Free Will, PPR 2016, S. 1 ff.; Fogal, Descartes, S. 1 ff.

20 So etwa Gilbert, Pac. Philos. Q. 86 (2005), S. $201 \mathrm{ff}$. 


\title{
I. Wille in den Anfängen seiner Philosophie
}

\section{Verstand und Wille}

In seiner ersten philosophischen Abhandlung Regulae, die Descartes in 1619-1620 angefangen, dann unterbrochen und 1926-1928 wieder aufgenommen hat, ${ }^{21}$ äußert sich Descartes zur Rolle des Willens in seiner Erkenntnistheorie nur spärlich. Er geht nicht ins Detail. Dies lässt sich an der folgenden Textstelle aus der Regulae erkennen:

\begin{abstract}
"Wenn also jemand ernsthaft die Wahrheit aufspüren will, so darf er keine vereinzelte Wissenschaften wählen; sie sind nämlich alle miteinander verbunden und voneinander abhängig; sondern sei nur darauf bedacht, das natürliche Licht der Vernunft zu vermehren, nicht im diese oder jene Schulfrage zu lösen, sondern damit in den einzelnen Vorfällen des Lebens der Verstand dem Willen vorschreibe, was zu wählen sei."22 [Hervorhebung hinzugefügt]
\end{abstract}

In diesen Stellen bringt Descartes vordergründig seine Überzeugung von der Notwendigkeit einer Gesamtwissenschaft zum Ausdruck und positioniert sich gegen die in der damaligen Scholastik herrschende aristotelische Theorie der einzelnen und voneinander unabhängig existierenden Wissenschaftsdisziplinen. Hinsichtlich einer möglichen prima philosophia bzw. einer Metaphysik, die beim Erkunden der physikalischen Welt die erkenntnistheoretischen Grundlagen bereitstellen soll, verspricht sich Descartes von dem natürlichen Licht der Vernunft große Erfolge. Dabei hofft er, dass der Verstand den Willen in die sicheren Bahnen der einzig richtigen Vernunfterkenntnisse steuern wird. Vor dem Hintergrund kann man an dieser Stelle konstatieren, dass Descartes die Einheit in den physischen Wissenschaften durch den Vorrang des Verstandes, der den hinsichtlich seiner Wahl unklaren Willen nur bessert, erreichen möchte. Deshalb wird ihm in der Literatur unter Verweis auf diese Überlegungen teilweise vorgeworfen, dass er - jedenfalls in seiner frühen Philosophie - einen Verstandsprimat postuliert und von einer intellektuellen Determiniertheit des Willens durch den Verstand ausgeht. ${ }^{23}$

Bei näherem Hinsehen erweist sich diese Schlussfolgerung an dieser Stelle als verfrüht. Denn Descartes spricht hier weder die genannte Problematik direkt an, noch vermag der Wortlaut des Textes eine solche Interpretation zu begründen. Dieser lässt nämlich nur die Schlussfolgerung zu, dass der Wille durch Vernunfterkenntnisse bestimmbar ist. ${ }^{24}$ Auf die Frage, ob sich der Wille dann trotzdem anderweitig entscheiden kann, geht Descartes nicht ein. ${ }^{25}$ Nichtdestotrotz stellt er hier zielorientiert den Idealfall für die prima philosophia seiner Gesamtwissenschaft dar, nämlich dass der Wille dem Verstand folgt. Die philosophisch eher

21 Hierzu siehe Perler, Descartes, S. 267.

22 AT X, 361.

23 So etwa Jayasekera, Ergo 3/2016, S. 321; Kahl, Die Lehre vom Primat des Willens, S. 114 f.

24 Hierzu siehe unten III.1. und 3.

25 Hierzu siehe unten III.1. und 3. 
interessantere Frage jedoch, wie der Wille beschaffen ist ${ }^{26}$ und ob er indeterminiert und selbstbestimmend ist, ${ }^{27}$ tastet er nicht an.

Wie bereits oben betont, ist Descartes in der Regulae vielmehr darum bemüht, seine Leser von der praktischen Notwendigkeit aber auch von der metaphysischen Möglichkeit einer Gesamtwissenschaft zu überzeugen, der eine disziplinenübergreifende Methode der Erkenntnisgewinnung zugrunde liegt, und in der disziplinendurchbrechende universale und absolute Wahrheiten gelten. ${ }^{28}$ Im Zentrum dieser Überlegungen von Descartes steht die Vernunfterkenntnis, die er an der folgenden Stelle der Regulae weiter in Gestalt einer mathesis universalis zur Erreichung der oben genannten Zwecke entwerfen möchte. Descartes führt aus:

"Denn es gibt kaum eine Frage in den Wissenschaften, über die scharfsinnige Leute nicht oft uneinig gewesen wären. Sooft indessen zweier Urteile über dieselbe Sache in die entgegengesetzte Richtung laufen, hat sich sicherlich mindestens einer von beiden getäuscht, und es besitzt offenbar nicht einmal einer von ihnen Wissenschaft. Wenn nämlich dessen Grund zuverlässig und evident wäre, so könnte er ihn dem anderen so auseinandersetzen, dass er zuletzt auch dessen Verstand überzeugt. .... Es folgt also, wenn unsere Rechnung stimmt, dass allein Arithmetik und Geometrie unter den bereits gefundenen Wissenschaften übrigbleiben, auf die uns die Beobachtung dieser Regel zurückführt." ${ }^{29}$ [Hervorhebung hinzugefügt]

Bei der zitierten Stelle wird Descartes hinsichtlich der Vernunfterkenntnisse, denen der Wille nach seinen Vorstellungen folgen möge, deutlicher. Ihm geht es darum, zu wissenschaftlichen Wahrheiten zu gelangen, die von einer absoluten Überzeugungskraft sind. Er glaubt daran, dass niemand solchen Wahrheiten seine Zustimmung verweigern kann, wenn diese mathematischer Natur sind. Das Problem der uneinheitlichen und unsicheren Wissenschaften kann nach Descartes nur so gelöst werden.

Im weiteren Verlauf seiner Ausführungen nimmt Descartes Bezug auf Erkenntnisse von anderer Art, bei denen der Wille dem Verstand in Anbetracht der fehlenden mathematischen bzw. deduktiven Exaktheit nicht von sich aus folgt. Er ist nämlich überzeugt davon, dass Intuition auch einige grundlegende Erkenntnisse liefern kann, denen der Wille nicht ausweichen kann. Diesbezüglich wird er in der Regulae wie folgt konkreter:

\footnotetext{
"Unter Intuition verstehe ich ...: eines reinen und aufmerksamen Geistes unbezweifelbares Begreifen, welches allein dem Lichte der Vernunft entspringt und .... So kann jeder intuitiv mit dem Verstand sehen, dass er existiert, dass er denkt, dass ein Dreieck von nur drei Linien, dass die Kugel von einer einzigen Oberfläche begrenzt ist. ${ }^{\text {“30 }}$ [Hervorhebung hinzugefügt]
}

26 Hierzu siehe unten II.1.

27 Hierzu siehe unten III.1. und 3.

28 Hierzu siehe auch Perler, Descartes, S. 51.

29 AT X, 363.

30 AT X, 368; für den Begriff des reinen Geistes im Zusammenhang mit der Intuition siehe ausführlich Perler, Descartes, S. $53 \mathrm{ff}$. 
Bemerkenswert ist bei dieser Stelle, dass Descartes zwar von einem aufmerksamen Geist spricht, der Tätigkeit der Aufmerksamkeit jedoch dem Licht der Vernunft gegenüber keine eigenständige Bedeutung zukommt. ${ }^{31}$ Denn Letzteres bildet für ihn die Ursache der intuitiven Erkenntnisse, dass er denkt und existiert. In seiner späteren Philosophie wird sich Descartes der Aufmerksamkeit noch näher widmen müssen, zumal dieses Kriterium einen selektiven und somit voluntativen Gehalt und deshalb eine deutliche Nähe zum Willen aufweist. ${ }^{32}$

\title{
2. Glaube - Wille und Gott
}

In der Regulae spielt der Wille erst dann eine eigenständige Rolle, wenn Descartes den Bereich der Erkenntnisse verlässt, die die physische Welt und deren Erfassen betreffen. Das dualistische Weltbild, das sich nicht nur durch die ganze Erkenntnistheorie von Descartes zieht, ${ }^{33}$ bringt kosmische und sozialpraktische Folgen mit sich, die über die Naturwissenschaften und die prima philosophia hinausgehen. Es ermöglicht ihm, der Religion und der Kirche einen Bereich zuzuweisen, in dem nicht verstandsmäßige Erkenntnisse gelten, sondern der Glaube bzw. der Wille herrschen. Folgende Textstelle aus der Regulae belegt das:

\begin{abstract}
"Was jedoch nicht hindert, dass wie das, was von Gott offenbart ist, für zuverlässiger als alle Erkenntnis halten, weil der Glaube daran, so sehr er auch auf Verborgenes geht, doch keine Tat der Erkenntniskraft, sondern des Willens ist,..." ${ }^{34}$ [Hervorhebung hinzugefügt]
\end{abstract}

Mit dieser Stelle macht Descartes klar, dass sein Interesse, verlässliche und sichere Erkenntnisse durch eine mathesis universalis $\mathrm{zu}$ erreichen und eine entsprechende Erkenntnistheorie zu entwerfen, grundsätzlich den Naturwissenschaften gilt. ${ }^{35}$ Auch wenn der Geist, dessen ontologische Existenz bereits in seiner dualistischen Metaphysik vorausgesetzt wird, bei Erfassen der physikalischen Wahrheiten über die Natur die entscheidende Rolle spielt, so schöpft er sich darin nicht aus. ${ }^{36} \mathrm{Er}$ ist für Descartes auch zugänglich für die Annahme der göttlichen Offenbarung. Der Wille vermag also den Glauben an göttliche Offenbarung zustande zu bringen.

Des Weiteren führt Descartes in der Regulae mit folgender Textstelle an, dass der Geist eine göttliche Natur hat:

31 Für eine neue Interpretation der Aufmerksamkeit in der cartesianischen Willenslehre siehe Schulte, Willensfreiheit und Aufmerksamkeit bei Descartes, ZPhF 71/1-2017, S. $18 \mathrm{ff}$.

32 Hierzu siehe unten II.3. und III.3; vgl. Kahl, Die Lehre vom Primat des Willens, S. 119.

33 Hierzu siehe auch Jaspers, Descartes und die Philosophie, S. 51 und 79.

34 AT X, 370.

35 CSMK, 234 (AT IV, 117 f.); hierzu siehe auch Perler, Descartes, S. 38 f.

36 Diesbezüglich siehe etwa auch folgende Stellen aus Descartes' Werk Beschreibung des menschlichen Körpers:

"Our volitions, in turn, are of two sort. One consists of the actions of the soul which terminate in the soul itself, as when we will to love God or, generally speaking, to apply our mind to some object which is not material. The other consists of actions which terminate in our body, as when our merely willing to walk has the consequence that our legs move and we walk.” (CSM I, 335; AT XI, 343). [Hervorhebung hinzugefügt]. 


\begin{abstract}
"Es besitzt nämlich der menschliche Geist etwas irgendwelche Gotterfülltes, worein die ersten Samen nützlicher Gedanken so gestreut sind, dass sie .... ${ }^{\text {"37 }}[$ Hervorhebung hinzugefügt]
\end{abstract}

Diese Stelle führt vor Augen, dass Descartes nicht nur antizipiert, dass der Mensch im Reich des theistischen Gottes ein Geist ist, sondern auch dass der Gott seinem Geist die ersten Ideen gegeben hat, ${ }^{38}$ damit er die ebenfalls von Gott geschaffene physische Welt überhaupt wahrnehmen kann. ${ }^{39}$ Später wird er in den Meditationen diese Ideenlehre fortentwickeln. ${ }^{40}$

Diese im Grundsatz egalitäre Einstellung Descartes zur Fähigkeit und Ausstattung aller Menschen, anhand der ihnen von Gott gegebenen ersten Gedanken zur universalen Wahrheit zu gelangen, begegnet einem praktischen Problem, das der eingangs geschilderten Fragestellung ähnelt: wenn alle Menschen die Fähigkeit haben, die Wahrheit zu finden, wie kommt es dann dazu, dass die Menschen über Dinge mit sich voneinander wesentlich abweichenden Ergebnissen urteilen?

\title{
3. Richtige Anwendung des Verstandes und Verantwortlichkeit des Gottes
}

Zu dieser Frage äußert sich Descartes in dem Discours de la méthode wie folgt:

“... die Verschiedenheit der Meinungen ... davon kommt, ...,..dass wir mit unseren
Gedanken verschiedene Wege verfolgen und nicht dieselbe Dinge betrachten. Denn es
kommt nicht bloß auf den gesunden Verstand, sondern wesentlich auch auf dessen
gute Anwendung an." 41 [Hervorhebung hinzugefügt]

Descartes benennt hier zwar den Grund der Meinungsverschiedenheiten, die auch die Falschheit einiger diesbezüglich gefällter Urteile impliziert, nämlich eine schlechte Anwendung des Verstandes. Wie sich der Geist im Einzelnen irrt, insbesondere ob der Irrtum dabei nicht nur wesentlich, sondern ausschließlich durch den Verstand zustande kommt, lässt er aber offen. Gleichzeitig weist die Anwendung des Verstandes auf das Vorliegen eines anderen Denktaktes hin, der von der Tätigkeit des Verstandes getrennt ist. Descartes geht aber an dieser Stelle nicht so weit, dass er hier den Grund der falschen Anwendung des Verstandes, wie er später in den Meditationen vornehmen wird, ${ }^{42}$ in der Beteiligung eines voreiligen Willens sieht. Im Gegensatz dazu ist Descartes in dem Discours de la méthode davon überzeugt, dass der Wille eigentlich die Neigung hat, dem Verstand zu folgen. Das bringt er wie folgt zum Ausdruck:

"...Denn unser Wille verlangt nur nach Dingen, die ihm der Verstand als in einer Art erreichbar darstellt...."43

37 AT X, 373.

38 Hierzu siehe auch Perler, Descartes, S. 55.

39 Für die Rolle des Gottes in der Erkenntnistheorie Descartes siehe mehr unten II.1.

40 Dazu siehe AT VII, $37 \mathrm{ff}$.

41 AT VI, 2.

42 Hierzu siehe unten II.2.

43 AT VI, 25. 
"...Denn wenn unser Wille nur das begehrt und vollzieht, was der Verstand ihm gut lehrt,...." ${ }^{\prime 4}$

Des Weiteren befasst sich Descartes bereits in dem Discours de la méthode mit der Frage, ob Gott für die Irrtümer, die Menschen, also seine Geschöpfe, begehen, verantwortlich gemacht werden kann. Bevor er darauf eingeht, unterstreicht er erneut wie folgt, dass Gott das Herzstück seiner gesamten Erkenntnistheorie ist:

"...Alles, was ich klar und deutlich erkenne, wahr sei, ist nur zuverlässig, weil Gott ist oder besteht, und weil er ein vollkommenes Wesen ist, und weil Alles in uns von ihm kommt; hieraus folgt, dass unsere Vorstellungen oder Begriffe als wirkliche Dinge, die, soweit sie klar und deutlich sind, von Gott kommen, wahr sein müssen." ${ }^{45}$ [Hervorhebung hinzugefügt]

Gott ist für Descartes also Garant absoluter und ewiger Wahrheiten und zwar in dreierlei Hinsicht: Gott hat alle Menschen dergestalt erschaffen, dass sie die ersten Gedanken und den Verstand haben und sich auf die Wahrheit der Gedanken verlassen dürfen, die klar und deutlich sind. ${ }^{46}$ Später in den Meditationen wird Descartes die Vollkommenheit des Gottes in diesem Zusammenhang weiter dahingehend ausführen, dass er die Menschen in der Erlangung der Wahrheit nicht täuschen würde. ${ }^{47}$

Die unzertrennlichen Verflechtungen zwischen seiner Erkenntnistheorie und den Eigenschaften des theistischen christlichen Gottes werfen die Frage auf, ob ein solcher Gott überhaupt existiert. Dieser widmet sich Descartes später in den Meditationen ausführlich. ${ }^{48}$ Die Problematik, dass Gott aber nicht nur für das Zustandekommen absoluter und ewiger Wahrheiten sorgt, sondern auch bei der eingangs vorgestellten Fragestellung bezüglich der Ursache des menschlichen Irrtums in Mitleidenschaft gezogen werden kann, bemerkt Descartes bereits in dem Discours de la méthode. Zwar unterlässt er hier, auf den Irrtum als eine rein erkenntnistheoretische Fragestellung einzugehen. Den theologischen Charakter des Irrtums kann er nicht unberührt lassen. Auf den Punkt gebracht stellt sich in diesem Zusammenhang insbesondere die Frage: ist die Ursache des Irrtums Gott selbst, indem er die Menschen irrtumsanfällig erschaffen hat? ${ }^{49}$

Zur Entlastung Gottes bringt er in dem Discours de la méthode folgende Argumente ein:

\footnotetext{
"Wenn wir also auch falsche Vorstellungen haben, so können es nur die verworrenen und dunklen sein; denn insoweit nehmen sie an dem Nichts Teil, d.h. sie sind nur deshalb in uns verworren, weil wir nicht ganz vollkommen sind." ${ }^{50}$ [Hervorhebung hinzugefügt]
}

44 AT VI, 28.

45 AT VI, 38.

46 Hierzu siehe auch Jaspers, Descartes und die Philosophie, S. 17.

47 Hierzu siehe unten IV.

48 AT VII, 45, 51-52 und 64.

$49 \mathrm{Zu}$ dieser Fragestellung siehe auch AT VIIIA, 19.

50 AT VI, 39. 
Für Descartes begehen die Menschen Irrtümer, weil sie nicht vollkommen sind. Da die Irrtümer aber nur epistemisch begangen werden, aber ontologisch gesehen keine Existenz haben, findet Descartes unmöglich, diese Gott zuzuschreiben. Bemerkenswert ist es, dass Descartes offenlässt, in welcher Weise die Unvollkommenheit der Menschen zu Irrtümern führt. Erst in den Meditationen vervollständigt er seine These dadurch, dass der menschliche Verstand beim Erfassen der Wahrheit begrenzt ist, daher nicht alles klar und deutlich sehen kann und der Wille die eigentliche Ursache eines Irrtumes ist, weil Letzterer trotz des Fehlens einer klaren und deutlichen Idee seine Zustimmung erteilt, obwohl es dem Willen möglich ist, sich in solchen Fällen zu enthalten. ${ }^{51}$

\section{Zwischenergebnisse}

Aus dem oben Gesagten lässt sich ableiten, dass sich Descartes in der Regulae und dem Discours de la méthode weder mit der metaphysischen Ursache der Irrtümer des Geistes noch mit dem Willen als solchem einschließlich der Frage seiner Freiheit auseinandergesetzt hat. Vielmehr wünscht er sich, dass der Wille dem Verstand folgt und dadurch in das Reich der klaren und deutlichen Ideen der absoluten Wahrheiten gelangt. Eine solche Neigung im Willen stellt Descartes ebenfalls fest. Unbestreitbare, absolute und ewige Wahrheiten, die der reine und aufmerksame Geist durch den Verstand erreichen kann, sind für Descartes nicht nur die Grundlagen der Mathematik, sondern auch Grundsätze einer dualistischen und intuitiven Philosophie des Geistes, etwa die Gewissheit über die eigene Existenz und die Natur der menschlichen Existenz als denkendes Ding. Der Wille spielt in seinen frühen Werken eher im spirituellen Bereich der menschlichen Existenz eine Rolle. Der theistische Gott hat für Descartes jedoch nicht nur die Offenbarung den Menschen zuteil werden lassen, sondern er ist auch Garant für absolute ewige Wahrheiten. Irrtümer, die der menschliche Geist begeht, sind aber keineswegs ihm zuzuschreiben.

Von diesen Thesen rückt Descartes in seinem späteren Werk Meditationen nicht ab. Er ergänzt und führt sie in einer neuen Darstellung an, wobei in der Regulae und dem Discours de la méthode die Kritik der herkömmlichen aristotelischen Wissenschaftstheorie, die ersten Gedanken einer prima philosophia und auch eine gewisse Euphorie im Vordergrund stehen. Gleichzeitig zeigen die oben vorgenommenen Ausführungen, dass der Wille in Descartes' Philosophie in zwei Zusammenhängen eine Bedeutung erhält: in seiner Erkenntnistheorie sowie in der Verbindung seiner prima philosophia mit Gott. Entsprechend dieser Unterscheidung wird unten zuerst der Wille im Rahmen seiner Erkenntnistheorie näher beleuchtet (Teil II. und III.) und dann mit der Idee Gottes in Beziehung gesetzt (Teil IV. und V.).

\section{Ursache des Irrtums}

\section{Beschaffenheit des Geistes und seiner Bestandteile}

Wie oben bereits festgestellt, geht Descartes in den Meditationen im Vergleich zu seinen früheren Werken systematischer vor. Nachdem er seine rationalistische Erkenntnistheorie und

51 Hierzu siehe mehr unten II.2. 
die Existenz Gottes in den Zweiten und Dritten Meditationen dargelegt und bewiesen haben will, befasst er sich mit der Frage, die der vorliegende Beitrag eingangs gestellt hat: wie und warum irrt sich der Geist?

Um Descartes' Lösungsansatz besser zu verstehen, lohnt es sich, zunächst auf einen Blick darauf zu werfen, wie Descartes den menschlichen Geist im Wesentlichen versteht.

In seinem Brief v. Mai 1641 an Regius führt Descartes an:

"There is only one soul in human beings, the rational soul: for no actions can be reckoned human unless they depend on reason. The vegetative power and the power of moving the body, which are called the vegetative and sensory souls in plants and animals... because they are not the first principle of their action, and they belong to a totally different genus from the rational soul"s2 [Letzte Hervorhebung hinzugefügt]

Aus dieser Stelle geht hervor, dass Descartes die Natur des menschlichen Geistes auf ein einziges Prinzip reduziert, nämlich die Rationalität/Vernunft, ${ }^{53}$ und die Handlungen des menschlichen Geistes allein im Lichte dieses Prinzips bewerten möchte. ${ }^{54}$ Ihn interessiert also der menschliche Geist, sofern die Gründe der Vernunft ihm zugänglich ist. Wenn sich Descartes in der Vierten Meditation den Ursachen der Irrtümer des menschlichen Geistes widmet, so hat er das Bild vor Augen, in dem sich der Geist in einer besonderen Konstellation der Gründe befindet, die ihn nicht zur Wahrheit, sondern zur Falschheit führt. ${ }^{55}$

Nach dem Nachweis der Existenz des Geistes in der Zweiten Meditation beschreibt Descartes dessen Natur dahingehend, dass dieser ein wollendes und erkennendes Ding ist. ${ }^{56}$ In der Vierten Mediation ist er sich auch sicher, dass der Geist ein Urteils- und Erkenntnisvermögen hat. ${ }^{57}$ Den epistemischen Gang der Irrtümer des Geistes im Rahmen der Anwendung der beiden Vermögen, ${ }^{58}$ der schlussendlich zur Falschheit führt, umreißt Descartes in den Vierten Meditation wie folgt:

"meine Irrtümer ..., dass sie von zwei gleichzeitig zusammenwirkenden Ursachen abhängen, nämlich vom Erkenntnisvermögen, das in mir ist, und von dem Vermögen zu wählen, bzw. von der Freiheit der Willkür, will sagen; vom Verstand und zugleich vom Willen. ".59 [Hervorhebung hinzugefügt]

52 CSMK, 182 (AT III, 371).

53 Hierzu siehe auch Gilbert, Pac. Philos. Q. 86 (2005), S. 215.

54 Zur Infragestellung der aristotelischen Seelenlehre durch Descartes siehe auch Perler, Descartes, S. 171.

55 In diesem Zusammenhang siehe etwa seinen Brief v. 2 Mai 1644 an Mesland, in dem Descartes argumentiert, dass die Tiere keinen Willen haben, weil sie keine Vernunft besitzen. So schreibt er:

"As for animals that lack reason it is obvious that they are not free, since they do not have this positive power to determine themselves; what they have is a pure negation, namely the power of not being forced or constrained". (CSMK, 234 (AT IV, 117).

56 AT VII, 28.

57 AT VII, 53 und 57; siehe auch Schulte, Willensfreiheit und Aufmerksamkeit bei Descartes, ZPhF 71/1-2017, S. 7.

58 In dem Discours de la méthode unterstrich Descartes noch die richtige Anwendung des Verstandes. Hierzu siehe oben I.3.

59 AT VII, 57; für das Zusammenwirken des Verstandes und des Willens in einem Urteil siehe auch Descartes Werk Prinzipien der Philosophie CMSK, 204 (AT VIIIA, 18). 
Bevor auf Descartes' Erklärung eingegangen wird, in welcher Weise sich dann der Wille und Verstand verhalten, sodass die Irrtümer entstehen, sei an dieser Stelle einige Anmerkungen über den Willen und den Verstand gemacht. So schreibt Descartes in seinem Brief v. 25. Dezember 1639 an Mersenne über den Willen wie folgt:

"The desire that everyone has to possess every perfection he can conceive of... is due to the fact that ... a will which has no limits" ${ }^{60}$ [Hervorhebung hinzugefügt]

Nach Descartes hat der Wille also eine innere Beschaffenheit, die darin besteht, das Vollkommene zu suchen und auch zu erlangen. ${ }^{61}$ In den Meditationen setzt er das auch mit dem Guten gleich. Er führt aus:

"Der Wille eines denkenden Dings geht .... unfehlbar auf das von ihm klar erkannte Gut. Wenn ihm daher irgendwelche Vollkommenheiten bekannt werden, die ihm fehlen, wird er sie sich unverzüglich verschaffen, wenn sie seinem Einfluss unterliegen". ${ }^{62}$ [Hervorhebung hinzugefügt]

Soweit dies die Natur des Willens ist, so kommt nach dem cartesianischen Verständnis ihm der Verstand zur Hilfe, indem er bei seiner Suche nach der Vollkommenheit bzw. dem Guten die Materie beleuchtet, die der Gegenstand des Willens ist ${ }^{63}$ bzw. auf die sich der Wille bezieht. ${ }^{64}$ In seinem Brief v. 24 Mai 1640 an Regius führt Descartes in diesem Zusammenhang an, dass der Geist nichts anders kann als dem zuzustimmen, was er klar erkennt:

"For our mind is of such a nature that it cannot help assenting to what it clearly understands..."

\section{Erkenntnisakt des Geistes}

Zu den inneren Handlungen des Geistes, die ihm wesenhaft sind, nämlich verstehen und wollen, ${ }^{66}$ äußert sich Descartes in seinem Brief v. Mai 1641 an Regius weiter. Im Hinblick auf ihr Zusammenwirken und ihr Verhältnis zueinander sagt er:

"..They differ only as the activity and passivity of one and the same substance. For strictly speaking, understanding is the passivity of the mind and willing is its activity: but because we cannot will anything without understanding what we will, and we scarcely ever understand something without at the same time willing something, we do not easily distinguish in this matter passivity from activity". ${ }^{67}$ [Hervorhebung hinzugefügt]

60 CSMK, 141 f. (AT II, 628); hierzu siehe auch Gilbert, Pac. Philos. Q. 86 (2005), S. 216.

61 Siehe auch Kahl, Die Lehre vom Primat des Willens, S. 122.

62 AT VII, 166.

63 Hierzu siehe etwa AT VII, 147.

64 Hierzu siehe etwa AT VII, 37; ähnlich auch AT VII, 28 und 58.

65 CSMK 147 (AT III, 64).

66 Hierzu siehe ausführlich Jaspers, Descartes und die Philosophie, S. 26 f.; Fogal, Descartes, S. 2.

67 CSMK, 182 (AT III, 372) 
Auf den ersten Blick erscheinen einem Descartes' Aussagen zwar verwirrend. Bei einem aufmerksamen Lesen lassen sich aber aus diesen wichtige Aufschlüsse darüber entnehmen, wie der cartesianische Wille und Verstand in einem Erkenntnisakt interagiert. Descartes' Grundannahme bezüglich des Willens ist, dass in einem Erkenntnisakt des Geistes dem Willen die aktivierende und entscheidende Rolle zukommt. Dabei unterscheidet er zwischen zwei Eigenschaften des Willens und setzte diese in Beziehung zum Verstand: der Wille kommt in seiner frühesten Form, die dem Geist bewusst wird, als Begehren vor. Bezieht er sich dabei auf etwas, so wird der Verstand aktiviert. Der Verstand erfasst also nichts, solange der Wille ihn nicht bewegt, weil Ersterer ja von seiner Natur aus passiv und Letzterer aktiv ist. Gleichzeitig unterscheidet Descartes zwischen dem begehrenden Willen, der sich auf etwas bezieht und den Verstand aktiviert, von dem entscheidenden Willen, der aus Urteilskraft besteht. Der Wille entschließt sich dann zu einem Urteil bezüglich eines Gegenstandes, den er anfangs begehrte und der Erfassung des Verstandes anheimstellte. ${ }^{68}$ Der Entschluss des Willens hat für Descartes im Ergebnis immer ein voluntatives und ein kognitives Element. ${ }^{69}$

Um die Irrtümer des Geistes zu erklären, braucht Descartes an dieser Stelle nicht zu begründen, warum der Wille teils aus einem fast unaufhörlichen Fluss des Begehrens im Bewusstsein besteht. ${ }^{70} \mathrm{Ihm}$ geht es vordergründig um die Rolle des entscheidenden Willens, der sich gegenüber Gründen befindet, die ihm der Verstand womöglich über die bloße kognitive Erfassung seines Gegenstandes hinaus präsentieren wird. Der Wille wird dabei zwar durch die Überzeugungskraft der Gründe hin und her bewegt, ${ }^{71}$ er bewahrt aber dabei weiterhin seine Kraft, sich für eine oder die andere Seite zu entschließen.

Wenn man sich die Funktionsweise einer solchen Theorie der voluntativen und kognitiven Elemente eines jeden Erkenntnisaktes vergegenwärtigt, ${ }^{72}$ so lassen sich je nach Stärke bzw. Schwäche dieser Elemente folgende Konstellationen herausarbeiten, ${ }^{73}$ in denen der Wille ein Urteil fällt und sich womöglich irren kann:

1. Der entscheidende Wille bringt beachtliche Anstrengungen zustande, um ein Urteil zu fällen, weil er bestimmte Ziele verfolgt und die kognitiven Grundlagen ihn deswegen weniger interessieren. Die voluntative Aufstockung kompensiert dabei die kognitive Schwäche.

68 Als weiteren Beleg für diese Unterscheidung kann etwa die folgende Stelle angeführt werden:

„...Denn wenn unser Wille nur das begehrt und vollzieht, was der Verstand ihm gut lehrt,..." [Hervorhebung hinzugefügt] AT VI, 28.

69 Vgl. Perler, Descartes, S. 162; Jayasekera, Ergo 3/2016, S. 325; Haag, Descartes über Willen und Willensfreiheit, ZPhF 60/4-2006, S. 485 ff.

70 Zum Begriff des Bewusstseins bei Descartes siehe Jaspers, Descartes und die Philosophie, S. 12.

71 Davon ausgehend ist er etwa auch bei der Aussage:

„Es muss außerdem darauf hingewiesen werden, dass die Klarheit, bzw. Transparenz, durch die unser Wille zur Zustimmung bewegt werden kann..." [Hervorhebung hinzugefügt] AT VII, 147.

72 Auffällig ist es, dass eine solche Theorie des inneren Handelns des menschlichen Geistes in den Rechtswissenschaften ziemlich gängig ist. Hierzu siehe etwa die Vorsatztheorie und Vorsatzformen im Strafrecht, Rengier, Allgemeiner Teil, S. 99 ff.

73 Diese Konstellationen wurden grundsätzlich im Lichte der im Strafrecht allgemein anerkannten Vorsatzformen entwickelt. 
2. Der entscheidende Wille unterliegt weder starken Entscheidungsmotiven noch verfügt er über sicheres Wissen, und:

a) er hält sich trotzdem davon nicht ab, ein Urteil zu fällen, oder

b) er enthält sich eines Urteils.

3. Der entscheidende Wille verfügt über sicheres Wissen und braucht vergleichsweise wenige Anstrengungen, um das Urteil zu fällen, weil der Wille bereits gewichtige Gründe für einen Entschluss hat.

In der Tat ergibt sich beim näheren Hinsehen, dass Descartes bei der Erklärung, wie der Wille sich irrt, wohl diese Konstellationen vor Augen hatte. So liegt es auf der Hand, dass sich der Wille in der 1. Konstellation höchst irrtumsanfällig verhält. Und tatsächlich irrt sich der Geist, der sich in dieser Situation befindet, immer wieder,

", weil der Wille weiter auslangt als der Verstand, [und ich ] ihn nicht in denselben Grenzen halte, sondern auch auf das ausweite, was ich nicht einsehe; da er diesbezüglich indifferent ist, weicht er leicht vom Wahren und Guten ab, und so täusche ich mich und gehe fehl." ${ }^{\text {74 }}$ [Hervorhebung hinzugefügt]

Der Wille erstrebt also etwas, obwohl aus der Sicht seines kognitiven Zustandes ungewiss ist, dass er zur Wahrheit gelangt. Der Wille begehrt zwar für Descartes immer das Wahre und das Gute, verfehlt es aber, weil er es nicht klar erkannt hat, deswegen indifferent ist und sich aus Gründen, die jedenfalls nicht verstandsmäßig sind, für etwas entschließt, das einen Irrtum enthält. ${ }^{75}$

Descartes erklärt wie folgt, warum die Indifferenz, die letztendlich zu einem Irrtum führt, mit einer gewissen Negativität behaftet ist:

"Jene Indifferenz aber, die ich erfahre, wenn kein Grund mich mehr zu der einen Seite als zu der anderen drängt, ist der geringste Grad der Freiheit und in ihr bezeugt sich keine Vollkommenheit, sondern lediglich ein Mangel an Erkenntnis,..." ${ }^{\text {“76 }}$

[Hervorhebung hinzugefügt]

Wie oben bereits festgestellt, besteht für Descartes die intrinsische Natur des Willens darin, nach Wahrheit zu suchen. Die Indifferenz, die der Wille erlebt, wenn er das Wahre nicht klar und deutlich erkennt, ist also insoweit zwar eine Freiheit, weil sich der Wille mit einem beachtlichen Kraftaufwand zu dem entschließt, was einen Irrtum enthält. Sie ist allerdings gleichzeitig der

74 AT VII, 58; siehe auch folgende Stelle:

„Diese Indifferenz erstreckt sich ja sogar über das hinaus, von dem der Verstand schlicht überhaupt nichts erkennt, allgemein auch auf alles, was von ihm zu derselben Zeit, zu der der Wille darüber einen Entschluss fasst, nicht hinreichend transparent erkannt wird. " (AT VII, 59).

75 Hierzu siehe auch Kahl, Die Lehre vom Primat des Willens, S. 118.

76 AT VII, 57. 
geringste Grad der Freiheit, weil der Wille keine klaren und deutlichen Gründe hat, sich von der Dunkelheit der Materie nicht befreien kann und sich der eigentlich auf das Gute gerichtete Wille im Ergebnis nur schwer entschließt. Auch die Konstellation in 2a) ist ein weiteres Bespiel, bei dem der Wille weiter auslangt als der Verstand. Obwohl er kein sicheres Wissen hat und seine Motive nicht stark sind, hält ihn die Vorstellung, dass er sich bei dieser Situation irren kann, nicht davon ab, ein Urteil zu fällen. Er findet sich mit der Idee eines Irrtums ab.

Erst in der Konstellation in 2b) wirkt die Indifferenz des Willens positiv, weil sich der entscheidende Wille, der die Kraft hat, das letzte Wort zu sagen bzw. das Urteil zu besiegeln, daran erinnert, dass er keinen Grund für die Erteilung seiner Zustimmung hat. ${ }^{77}$

Die 3. Konstellation ist für Descartes der Idealfall, in dem der Wille, der bereits entsprechend seiner inneren Bestimmung die Wahrheit sucht, diese nun mit sicheren Gründen erlangt. Die positive Freiheit ist bei Descartes also für den Fall reserviert, dass der Wille der klaren und deutlichen Idee, die ihm der Verstand vorlegt, spontan folgt und sich beim Entschließen voluntativ bzw. willentlich nicht anstrengen muss. Diese Gedanken bringt Descartes wie folgt zum Ausdruck:

\begin{abstract}
" je mehr ich zu der einen Seite neige ... weil ich auf dieser Seite den Grund des Wahren und Guten evident einsehe... desto freier wähle ich sie.... Wenn ich nämlich immer klar sähe, was das Wahre und das Gut ist, müsste ich niemals über das, was zu beurteilen oder zu wählen wäre, einen Entschluss fassen, und könnte so niemals indifferent, obwohl doch völlig frei sein. "78 [Hervorhebung hinzugefügt]
\end{abstract}

Frei ist der Wille nach Descartes also deswegen, weil der Wille, der grundsätzlich das Wahre und Gute begehrt, nun als Entscheidungsinstanz kaum eine Anstrengung braucht, um seine Zustimmung zu erteilen, wenn er über sichere Wissensgründe verfügt und dem Verstand spontan folgt. Die Freiheit, Spontanität und Willentlichkeit fallen für Descartes zusammen. ${ }^{79}$

\title{
3. Vermeidbarkeit der Irrtümer und Verantwortlichkeit der Person
}

Descartes interessiert sich nicht nur für die Frage, wie der Wille sich irrt und ob er dabei frei ist, sondern auch dafür, warum sich der Wille im Einzelnen irrt und ob der Geist das vermeiden

77 Das Paradebeispiel für diese neutrale Freiheit des Willens ist Descartes eigene Methode der sukzessiven Erkenntniserlangung durch ein striktes Zweifeln. Aus seiner Erfahrung bei den Meditationen berichtet, wie er bis zur Vierten Meditation zwar mit Gewissheit die Erkenntnis von seiner eigenen Existenz und von der des Gottes erlangt hat, aber als Geist noch nicht in die physische Welt eingedrungen ist.

„Jetzt aber weiß ich nicht nur, dass ich existiere, insofern ich ein gewisses denkendes Ding bin, sondern ich sehe mich auch der Idee einer gewissen körperlichen Natur gegenüber,... Setzte ich nun voraus, dass ich bislang meinem Verstand keine Begründung dargeboten habe, die mich mehr von dem einen als von dem anderen überzeugt hätte, so bin ich sicherlich schon allein deswegen indifferent, das eine oder das andere zu behaupten oder zu bestreiten, oder auch nichts über diese Sache zu urteilen." [Hervorhebung hinzugefügt] AT VII, 58.

78 AT VII, 57; ähnlich auch AT VII, 147.

79 Vergleiche dies mit III.3; siehe auch Haag, Descartes über Willen und Willensfreiheit, ZPhF 60/4-2006, S. 487 und 489 . 
kann. Beim näheren Hinsehen unterscheidet er zwischen zwei Arten von Irrtümern: diejenigen, die begangen werden, weil der menschliche Verstand begrenzt ist und nicht alles erfassen kann. Infolgedessen liefert er Ideen, an deren Klarheit und Deutlichkeit, also Wahrheit, der Wille fälschlicher-, aber wohlwollenderweise glaubt und seine Zustimmung erteil. Zur zweiten Art der Irrtümer zählt Descartes diejenigen, die der Wille unaufmerksam begeht, obwohl sie vermieden werden können. Dies erklärt Descartes wie folgt:

"..auch wenn ich mich nicht in der Weise vor Irrtümern bewahren kann, die von der evidenten Erfassung alles dessen abhängen, worüber ein Entschluss gefasst werden muss, ... obwohl ich die Unzulänglichkeit in mir erfahre, mich nicht unausgesetzt auf eine und dieselbe Erkenntnis konzentrieren zu können, so kann ich doch durch aufmerksame und oft widerholte Meditation bewirken, ... ${ }^{\text {"80 }}$ [Hervorhebung hinzugefügt]

Nach dieser Unterscheidung ist also nicht auszuschließen, dass sich der Geist auch in der oben genannten 3. Konstellation irrt, obwohl der Wille bei seinem Entschluss keinen Zweifel mehr hatte bzw. von der Gewissheit der verstandsmäßigen Gründe überzeugt war. Denn auch wenn der Wille gut ist und bei der Fällung des Urteils wohlwollend war, kann sich der Verstand nicht auf alles Wahre erstrecken. ${ }^{81}$ Allerdings kann hier auch die Unaufmerksamkeit des Willens verursacht haben, dass der Wille die genannte Gewissheit angenommen hat. In diesem Fall stellt sich die Frage, ob dies vermeidbar war oder nicht.

Diese Differenzierung ist außerdem für die Frage von Bedeutung, inwiefern die Person, die den freien Willen anwendet, für dessen Folgen zur Verantwortung gezogen werden kann. ${ }^{82}$ Während die Person für unvermeidbare Irrtümer von ihrer "epistemischen Schuld“ freizusprechen ist, darf ihr bei den Vermeidbaren der Vorwurf gemacht werden, dass sie durch einen unaufmerksamen Willen in ihrem "kognitiven Urteil" fehlgegangen ist, obwohl die Möglichkeit bestand, sich wenigstens wie etwa der Konstellation in 2b) zu enthalten..$^{83}$ Dabei macht Descartes klar, dass es für ihn stets und grundsätzlich auf den Imperativ der richtigen Anwendung der Urteilskraft ankommt. Gelangt eine Person durch die falsche Anwendung zufällig zum richtigen Ergebnis, so ändert das an der Tatsache nichts, dass der Geist dem Imperativ nicht gefolgt ist und deshalb weiterhin dafür verantwortlich gemacht werden kann. Mit Descartes Worten:

"Wenn ich aber nicht ausreichend klar und deutlich erfasse, was das Wahre ist, ist es klar, dass ich richtig handele und mich nicht täusche, ..., treffe ich zwar durch Zufall die Wahrheit, werde deshalb aber noch nicht der Verantwortung dafür entbunden sein: Denn es ist durch das Natürliche Licht offenkundig, dass

$80 \quad$ AT VII, 61.

81 Siehe diesbezüglich auch seinen Brief v. 6. Oktober 1645 an Prinzessin Elisabeth:

„..., it is true that we lack infinite knowledge which would be necessary for a perfect acquaintance with all the goods between which we have to choose in the various situations of our lives. We must, I think, be contented with a modest knowledge of the most necessary truths ..." [Hervorhebung hinzugefügt] (CSMK, 269; AT IV, 309).

82 Zur Verantwortlichkeit bei Descartes siehe ausführlich Jayasekera, Ergo 3/2016, S. 325 ff.

83 Zur Vermeidbarkeit eines Fehlers und "Schuld" des Geistes siehe auch Jaspers, Descartes und die Philosophie, S. 26. 
stets die Erfassung des Verstandes der Bestimmung des Willens vorhergehen muss. " ${ }^{44}$ [Hervorhebung hinzugefügt]

Des Weiteren lässt sich beim näheren Hinsehen feststellen, dass Descartes seine Erkenntnistheorie nicht nur als die richtige metaphysische Erfassung der Welt verstanden haben möchte, sondern auch als eine praktische Methode, mit deren Hilfe handfeste Ergebnisse erzielt werden können. Durch die Herausarbeitung der verschiedenen Konstellationen, in denen die voluntativen und kognitiven Elemente des Geistes unterschiedlich involviert sind, möchte Descartes dem Geist vor Augen führen, warum er sich irrt und wie er Irrtümer vermeiden kann. ${ }^{85}$ Der Geist soll sich die Einsicht einprägen, sich eines Urteils zu enthalten, solange er keine klare und distinkte Idee durch den Verstand vorgelegt erhalten hat. Sein Ideal ist, dass der Geist diese Einsicht im Gedächtnis

"durch aufmerksame und oft widerholte Meditation" fest verankert, und dass er "auf diese Wiese so etwas wie eine Gesinnung er[wirbt], [sich] nicht zu irren." [Hervorhebung hinzugefügt].

Nach dieser Einprägung im Gedächtnis und der entsprechenden Gesinnung des Geistes ist Descartes erstens davon überzeugt, dass die positive Freiheit des Willens ihm ermöglichen wird, sichere Erkenntnisse zu erlangen, und zweitens, dass die Freiheit des Willens ihn in die Lage versetzen wird, sich selbst nicht zu täuschen. ${ }^{87}$

Im Ergebnis geht es Descartes in der Vierten Meditation nicht lediglich um seine Einschätzung des Willens als ein metaphysisches Phänomen. Er braucht einige Ansätze mit Hand und Fuß, die ihm nicht nur bei der Grundlegung seiner Metaphysik zu verlässlichen und sicheren Ergebnissen führen, sondern auch von künftigen Wissenschaftlern in ihren empirischen Arbeiten herangezogen werden können.

\section{Zwischenergebnisse}

Die oben vorgenommenen Ausführungen haben gezeigt, dass sich Descartes' Willenstheorie nur vor dem Hintergrund einiger fundamentaler Grundannahmen der Philosophie des Geistes verstehen lässt. Der vorliegende Beitrag hat diesbezüglich folgende cartesianische Prämisse hinsichtlich des Willens herausgearbeitet:

i. Der rationale Mensch hat Urteils- und Erkenntnisvermögen, sodass er Dingen außer sich selbst wollen und verstehen kann.

ii. Der menschliche Wille ist auf das Gute gerichtet.

84 AT VII, 59.

85 Hierzu siehe auch Perler, Descartes, S. 163.

86 AT VII, 61.

87 Hierzu siehe AT VII, 62; zur Angewöhnung des Geistes, sich mit verstandsmäßigen Erkenntnissen zu begnügen und Irrtümer dadurch zu vermieden, äußert sich Descartes bereits in dem Discours, siehe AT VI, 26. 
iii. Der Wille ist die aktive Fakultät des Geistes und der Verstand ist die passive.

iv. Der Wille ist erstens Begehren und zweitens Entscheiden.

v. Der Wille kommt in seiner frühesten Form, die dem Geist bewusst wird, als Begehren vor.

vi. Bezieht er sich auf etwas, so wird der Verstand aktiviert.

vii. Der Wille entschließt sich dann zu einem Urteil bezüglich seines Gegenstandes, den er anfangs begehrte und der Erfassung des Verstandes anheimstellte.

Die Irrtümer des Willens sowie dessen Indifferenz sowie Freiheit lassen sich nur im Lichte dieser Grundannahmen nachvollziehen. Außerdem stellt Descartes einige Leitlinien auf für den Geist, sodass er Irrtümer vermeidet. Allerdings sind Descartes' Erklärungen über die Entstehung der Irrtümer des Geistes einschließlich der Rolle des Willens nicht nur meditative und praktische Richtlinien für eine richtige Anwendung des Urteilsvermögens. ${ }^{88}$ Sie betreffen darüber hinaus wichtige Fragen der Philosophie des Geistes. Das gilt allem voran dem Willen und seiner Freiheit, die Descartes in der Vierten Meditation annimmt. Bereits in den Einwänden gegen die Meditationen wird er mit einigen schwierigen Fragen konfrontiert, worauf er seine Willenstheorie weiter erklärend antwortet. Auch nach den Meditationen muss sich Descartes mit weiteren Fragen auseinandersetzen. Dabei modifiziert er seine Willenslehre in gewisser Hinsicht. Im Folgenden wird auf diese beiden Themenbereiche eingegangen.

\section{Cartesianische Willenslehre und ihre Fortentwicklung}

\section{Cartesianische Freiheit}

Wie die oben aufgeführten Erörterungen über das cartesianische Willensverständnis zeigen, geht Descartes in seiner Philosophie von einem finalen Freiheitsbegriff aus. Die Freiheit des Willens bedeutet also für ihn nicht, dass der Wille ein von jedem Zweck losgelöstes und aus dem Nichts und für eigene Zwecke kausal werdendes Vermögen ist. Im Gegensatz dazu ist der Wille intrinsisch auf das Wahre und Gute gerichtet. ${ }^{89}$ Er begehrt diese und folgt ihnen auch willentlich und frei, also spontan.

Descartes versucht in der Vierten Meditation zu erklären, was er unter der Freiheit des Willens versteht:

"Denn es besteht ja lediglich darin, dass wir dasselbe tun oder lassen können (will sagen: es behaupten oder bestreiten, es anstreben oder vermeiden können), oder vielmehr: es besteht lediglich darin, dass wir uns zu dem, was uns vom Verstand vorleget wird, um es zu behaupten oder zu bestreiten, bzw. anzustreben oder zu

88 Siehe diesbezüglich etwa seinen Brief v. 6. Oktober 1645 an Prinzessin Elisabeth: „Our nature is so constituted that our mind needs much relaxation if it is to be able to spend usefully a few moments in the search for truth." [Hervorhebung hinzugefügt] (CSMK, 268 f.; AT IV, 307).

89 AT VII, 166; AT VII, 431; hierzu siehe auch oben II.1. 
vermeiden, so verhalten können, dass wir empfinden, keine äußere Kraft bestimme uns zur Entscheidung. " ${ }^{\text {"90 }}$ [Hervorhebung hinzugefügt]

In der Literatur wird verschiedentlich zum Ausdruck gebracht, dass Descartes dadurch eine Definition der Freiheit des Willens einbringt. Die Interpretation dieser Stelle ist allerdings nicht leicht und führt in der Literatur zu Kontraversen. ${ }^{91}$ Das gilt allem voran der Tatsache, dass Descartes eine und dieselbe Definition mit zwei Formulierungen zu geben scheint, was gleich die Frage aufwirft, ob dann die beiden Definitionen gleichwertige Alternativen sind oder die eine dem anderen gegenüber Vorrang hat. Nicht nur die Vereinbarkeit der beiden Alternativen ist im ersten Fall ein schweres Unterfangen, ${ }^{92}$ sondern auch die Vorrangfrage lässt sich ohne Berücksichtigung der cartesianischen Willenslehre in ihrer Gesamtheit schwer beantworten. Diesem Definitionsstreit in der Literatur kommt bei Descartes Interpretationen insgesamt eine entscheidende Bedeutung zu, weil man sich hinsichtlich der Bewertung seiner Willenslehre als deterministisch, indeterministisch oder kompatibilistisch am Scheideweg befindet. ${ }^{93}$ Hinzukommt auch der Streitpunkt, ob Descartes im Hinblick auf das Vorrangsverhältnis zwischen dem Verstand und dem Willen in der Erlangung des Wahren oder des Guten als ein Vertreter des Intellektualismus oder des Voluntarismus einzuordnen ist. ${ }^{94}$

In der Tat spricht die erste Definition der Freiheit für einen indeterministischen Standpunkt, weil Descartes dem Willen das Vermögen zukommen lässt, sich in einem Fall alternativer Entscheidungsmöglichkeiten $\mathrm{zu}$ bedienen, nämlich etwas zu behaupten oder dasselbe zu bestreiten. Allerdings lassen sich andere Textstellen in der Vierten Meditationen finden, die dagegensprechen. Descartes stellt klar, dass er für die Freiheit des Willens nicht voraussetzt, dass sich dieser in allen Fällen, also ungeachtet des Grads der Evidenz der Gründe, hätte auch anders verhalten können. Dies bringt er wie folgt zum Ausdruck:

\section{"Um frei zu sein, ist es nämlich nicht nötig, dass ich mich nach beiden Seiten hin tatsächlich gleich verhalten kann." ${ }^{\text {"95 }}$ [Hervorhebung hinzugefügt]}

Diese cartesianische Position ist in der Tat mit der in der zeitgenössischen Philosophie immer wieder vertretenen Auffassung unvereinbar, dass die für die Bejahung der Freiheit des Willens als erste Bedingung eine Begründung für das sogenannte Prinzip alternativer Möglichkeiten (oder principle of alternative possibilities) verlangt. Hiernach setzt die Freiheit des Willens stets voraus, dass er sich innerhalb von mehreren alternativen Entscheidungsmöglichkeiten für eine von ihnen gleichermaßen entschließen kann. Mit anderen Worten bedarf die Bejahung der Willensfreiheit

90 AT VII, 57.

91 Hierzu siehe Fogal, Descartes, S. 1; Embry, Descartes on Free Will, PPR 2016, S. 1; Haag, Descartes über Willen und Willensfreiheit, ZPhF 60/4-2006, S. 483; Schulte, Willensfreiheit und Aufmerksamkeit bei Descartes, ZPhF 71/1-2017, S. 5.

92 Haag, Descartes über Willen und Willensfreiheit, ZPhF 60/4-2006, S. 484.

93 Hierzu siehe etwa Fogal, Descartes, S. 4 ff.

94 Hierzu siehe Wee, Descartes and Leibniz, CJPh 36/3-2006, 392.

95 AT VII, 57; ähnlich auch AT VII, 147. 
retrospektiv immer der hypothetischen Prüfung, dass sich der Wille auch hätte anders verhalten können. ${ }^{96}$

Ein gewisser intellektueller Determinismus wird Descartes unterstellt, wenn er immer wieder als idealen Fall des Gebrauchs der Willensfreiheit den Zustand hervorhebt, dass der Wille einer klaren und deutlichen Idee, die ihm der Verstand vorlegt, Folge leistet. ${ }^{97}$ Eine ähnliche Determiniertheit des Willens durch den Verstand lässt sich an folgender Stelle feststellen:

"...Als ich zum Beispiel in diesen Tagen prüfte, ob irgendetwas in der Welt existierte, und feststellte, dass allein daraus, dass ich dies prüfe, evident folge, dass ich existiere, konnte ich gar nicht umbin, zu urteilen, dass das, was ich so klar einsah, wahr sei; und zwar nicht, weil ich von irgendeiner äußeren Kraft dazu getrieben worden war, sondern weil sich aus dem großen Licht im Verstand eine starke Neigung im Willen ergeben hat, habe ich dies von selbst und frei umso mehr geglaubt, je weniger indifferent ich in bezug darauf gewesen bin. ${ }^{\text {"98 }}$ [Hervorhebung hinzugefügt]

Zwar impliziert hier das Fehlen einer alternativen Entscheidungsmöglichkeit für den Willen und die starke Neigung im Willen durch den Verstand eine Position, die man als intellektuellen Determinismus beim positiven Gebrauch der Willensfreiheit bezeichnen kann. ${ }^{9}$ Dieselbe Stelle kann jedoch unter dem Verweis auf das Fehlen äußerer Kräfte als ein kompatibilistisches Willensverständnis bei Descartes verstanden werden. ${ }^{100}$

Die oben zitierten Textstellen aus der Vierten Meditation sind nicht nur mit einer gewissen Ambiguität behaftet, ${ }^{101}$ sondern lassen auch eine eindeutige Einordnung der cartesianischen Willenslehre in der Terminologe der heutigen Willensdebatte kaum zu. Hinzukommt es, dass Descartes' Theorie zu seiner Zeit selbst mit seinen eigenen Prämissen nicht alle überzeugte.

\section{Kritik}

So wirft Hobbes im Dritten Einwand Descartes vor, dass er zwar die Freiheit des Willens annimmt, dafür aber keinen Nachweis erbracht hat. ${ }^{102}$ Daraufhin gibt Descartes eine recht einfache Antwort:

"Über die Freiheit habe ich hier nichts angenommen, außer dem, was wir alle in uns erfahren, und was für das Natürliche Licht ganz selbstverständlich ist." ${ }^{103}$ [Hervorhebung hinzugefügt]

96 Hierzu siehe Beckermann, s.1; Embry, Descartes on Free Will, PPR 2016, S. 1; siehe auch unten III.2. und 3.

97 Siehe auch Kahl, Die Lehre vom Primat des Willens, S. 119.

98 AT VII, 58.

99 Embry, Descartes on Free Will, PPR 2016, S. 3.

100 Hierzu siehe etwa Gilbert, Pac. Philos. Q. 86 (2005), S. 212 f.; Fogal, Descartes, S. 1; Wee, Descartes and Leibniz, CJPh 36/3-2006, 394; Schulte, Willensfreiheit und Aufmerksamkeit bei Descartes, ZPhF 71/1-2017, S.10.

101 So auch Kahl, Die Lehre vom Primat des Willens, S. $114 \mathrm{f}$.

102 AT VII, 190.

103 AT VII, 190. 
Als Antwort auf denselben Einwand führt Descartes außerdem an:

"...Dass willentlich und frei dasselbe ist, erfährtjeder, der nur sich selbst betrachtet. ${ }^{\text {104 }}$

Somit setzt Descartes zwar die von jedem erfahrene Freiheit mit der Willentlichkeit gleich. Er erklärt aber nicht, ob er damit den begehrenden Willen meint oder den Willen als Urteilskraft vor Augen hat. In Verbindung damit greift Hobbes Descartes mit folgenden Thesen an:

"... Sowohl zu wissen, dass etwas wahr ist, als auch etwas zu glauben oder ihm seine Zustimmung zu geben, hat nichts mit dem Willen zu tun. Denn was durch gültige Argumente nachgewiesen oder was glaubhaft berichtet wird, glauben wir, ob wir nun wollen oder nicht. Es ist wahr, dass Proportionen zu behaupten und zu bestreiten, zu verfechten und zurückzuweisen Akte des Willens sind; daraus folgt aber nicht, dass die innere Zustimmung vom Willen abhängt "105

Mit dem Argument stellt Hobbes die Rolle des begehrenden Willens in Frage. Er führt an, dass der Wille bei einem gültigen Argument sich darauf nicht richten oder es anstreben muss. Dagegen erkennt Hobbes die Rolle des entscheidenden Willens als Urteilskraft an, solange er etwas beschreitet oder verficht. Allerdings ist er der Auffassung, dass die innere Bestimmung des entscheidenden Willens unklar ist. Descartes antwortet auf diese Kritik durch die Wiederholung seiner eigenen Theorie:

"Wenn aber hier gesagt wird, dass wir nicht klar durchschauten Dingen zustimmen, ob wir nun wollen oder nicht, so ist das gerade so, ob wenn man sagen würde, dass wir ein klar erkanntes Gut begehren, ob wir nun wollen oder nicht; denn das Wort nicht wollen ist in diesem Zusammenhang fehl am Platze, weil es in sich widersprüchlich ist, dasselbe zu wollen und nicht zu wollen. " 106

Da der Wille nach dem cartesianischen Verständnis entsprechend seiner Natur von Beginn an das Gute begeht, setzt sich das Begehren des Willens fort, wenn der Verstand etwas Gutes klar erkannt hat. Wie bereits oben festgestellt, ist nach Descartes sogar die Willentlichkeit, die der entscheidende Wille bei der Erteilung seiner Zustimmung in so einem Fall erteilt, eben die Freiheit selbst. Insofern also Hobbes einen Akt des Willens bei der Erteilung der Zustimmung im Fall eines klar erkannten Dinges verneint sowie bei der Ablehnung einer Sache eine innere Involvierung des Willens in Frage stellt, so stößt er beim Descartes auf das Prinzip des auf das Gute gerichteten Willens, seiner Willentlichkeit und Freiheit bei einer klar erkannten Wahrheit und auf die Urteilskraft des ebenfalls auf das Gute gerichteten Willens, einer unklaren Sache seine Zustimmung zu verweigern. So wie Hobbes, bringt Descartes hier in der Sache keine neue Begründung seiner Prinzipien, die er für den Willen bereits in den Meditationen angenommen hat. 
Grundsätzlich bleibt Descartes bei seinem finalen Freiheitsverständnis. In seinen Erwiderungen auf den Sechsten Einwand bringt er erneut seine These zum Ausdruck:

\begin{abstract}
"Was hingegen den Menschen betrifft: Da er bereits eine von Gott bestimmte Natur alles Guten und Wahren vorfindet und seinen Willen gar nicht auf irgendetwas anderes richten kann, ist es evident, dass der Mensch desto williger und demnach freier das Gute und Wahre vertritt, je klarer er es sieht. ... Und schließlich gehört die Indifferenz nicht zum Wesen der menschlichen Freiheit. Denn wir sind nicht nur dann frei, wenn die Unkenntnis des Richtigen uns indifferent sein lässt, sondern vielmehr auch wenn eine klare Erfassung uns drängt, etwas anzustreben.“ ${ }^{107}$ [Hervorhebung hinzugefügt]
\end{abstract}

Es liegt auf der Hand, dass Descartes mit einer solchen Willenslehre, die sich in einem System der guten Zwecke bewegt, seine zeitgenössischen Philosophen, die aus einer scholastischen Tradition kamen, zwar mehrheitlich und ohne große Schwierigkeiten zu überzeugen vermochte. Nicht alle philosophischen Bedenken konnte er allerdings mit seiner Lehre ausräumen. Große Probleme bereitete insbesondere seine These, dass der Wille im Falle einer klar und deutlich erkannten Wahrheit dieser spontan folgt und seine Zustimmung nicht verweigern kann. Denn wie könnte der Wille frei sein, wenn er Letzteres nicht tun kann? Descartes hatte in den Meditationen die Freiheit des auf das Gute gerichteten Willens mit seiner Willentlichkeit in so einem Fall erklärt. Dies ändert allerdings nichts daran, dass die Freiheit des Willens im Ergebnis begrenzt bleibt. Auch Descartes' Argument, dass die Indifferenz nicht zum Wesen der menschlichen Freiheit gehört, klingt sehr apokalyptisch und lässt sich nur durch ein finales Freiheitsverständnis erklären.

In zwei Briefen an Mesland versucht Descartes einen Ausweg zu finden, sodass seine Willenslehre auch philosophischen Anforderungen genügt. ${ }^{108}$ Gleichzeitig ist er darum bemüht, die Modifikation so beschränkt zu halten, dass er seine Lehre nicht von Grund auf ändern muss oder diese dann nicht mehr konsistent ist. Im Folgenden wird die Fortentwicklung der cartesianischen Willenslehre im Lichte der beiden Briefe erörtert.

\title{
3. Modifikation des Freiheitsverständnisses
}

In seinem ersten Brief v. 2. Mai 1644 an Mesland zeigt Descartes eine Möglichkeit auf, bei der der Wille einer klar und deutlich erkannten Wahrheit doch nicht folgt. So führt er an:

".... For it seems to me certain that a great light in the intellect is followed by a great inclination in the will; so that if we see very clearly that a thing is good for us, it is very difficult - and, on my view, impossible, as long as one continues in the same thought - to stop the course of our desire. But the nature of the soul is such that it hardly

107 AT VII, 431; ähnlich auch AT VII, 166.

108 Zur Fortentwicklung der cartesianischen Willenslehre siehe auch Kahl, Die Lehre vom Primat des Willens, S. 122 f.; Schulte, Willensfreiheit und Aufmerksamkeit bei Descartes, ZPhF 71/1-2017, S. 6. 
attends for more than a moment to a single thing; hence, as soon as our attention turns from the reasons which show us that the thing is good for us, and we merely keep in our memory the thought that it appeared desirable to us, we can call up before our mind some other reason to make us doubt it, and so suspend our judgement, and perhaps even form a contrary judgement. ...." ${ }^{109}$ [Hervorhebung hinzugefügt]

Descartes Argument ist an dieser Stelle, dass der Wille zwar unmöglich davon abgehalten werden kann, das klar erkannte Gute zu wollen und einer entsprechenden Idee zuzustimmen. Dies erfolgt allerdings nach Descartes unter einer Bedingung, die er erst hier einbringt: solange sich der Wille dieselbe Idee anschaut. Denn dieser könne sich plötzlich von der Überzeugungskraft der entsprechenden Gründe abwenden, weil er seine Aufmerksamkeit nur schwer der einen und selben Sache geben könne. Daraus leitet Descartes ab, dass der Wille hinsichtlich derselben Idee also auch ein abweichendes Urteil fällen kann. ${ }^{110}$ Ist er damit von seiner früheren Ansicht in den Meditationen abgekehrt, dass die Indifferenz nicht zum Wesen der Freiheit gehört? ${ }^{111}$ In demselben Brief schreibt Descartes an Mesland, dass die Freiheit für ihn weiterhin die Willentlichkeit bedeutet und seine nun modifizierte Ansicht im Ergebnis doch mit der entgegengesetzten Auffassung übereinstimmt, die unter der Freiheit die Indifferenz des Willens bei seiner Selbstbestimmung in allen Fällen versteht. So führt er an:

\section{"I call free in the general sense whatever is voluntary, whereas you wish to restrict the term to the power to determine oneself only if accompanied by indifference. But as far as words are concerned, I wish above all to follow usage and precedent." 112 [Hervorhebung hinzugefügt]}

Auch wenn Descartes sagt, dass es sich hier lediglich um einen begrifflichen Unterschied handelt, so ist er weiterhin abgeneigt, undifferenziert eine Urteilskraft des Willens anzunehmen, die in einer allgemeinen Indifferenz des Willens bei seiner Selbstbestimmung bestehen würde. Denn ein solches Willensverständnis ist nicht nur dann mit dem cartesianischen finalen Freiheitsverständnis - auch nach der erwähnten Modifikation - schwer vereinbar, wenn der Wille eine klar und deutlich erkannte Idee vor sich hat. Eine Freiheit der Selbstbestimmung ist auch mit Descartes' Begründung des Irrtums unvereinbar. Denn für ihn erfolgt der Irrtum gerade deswegen, weil der Wille keine klare und deutliche Idee hat, deswegen indifferent, also nicht frei ist und trotzdem über den Verstand ,auslangt“ “. ${ }^{113}$ Er schließt also die Möglichkeit aus, dass der Wille klar und deutlich einsehen kann, dass er einen Irrtum begeht und sich dementsprechend entschließt. Nun ist zwar eine solche Konstellation für eine Willenslehre, die Descartes für eine prima philosophia der Naturwissenschaften bzw. eine Erkenntnistheorie konzipiert, von nicht großer Bedeutung. Descartes gerät allerdings mit seiner Indifferenzthese bei Irrtümern des

109 CSMK, 234 (AT IV, 116)

110 Hierzu siehe auch Wee, Descartes and Leibniz, CJPh 36/3-2006, 398; Kahl, Die Lehre vom Primat des Willens, S. $123 \mathrm{f}$.

111 Hierzu siehe oben III.2.

112 CSMK, 234 (AT IV, 116).

113 AT VII, 58. 
Geistes in Erklärungsschwierigkeiten, wenn es zu einem moralischen Irrtum bzw. einer Sünde kommt. Ist also der Mensch unfrei, wenn er sündigt? ${ }^{114}$ In seinem ersten Brief v. 2. Mai 1644 an Mesland muss Descartes auch dazu eine Stellung beziehen, obwohl er diesen Bereich eigentlich gar nicht betreten möchte. So schreibt er:

„The only thing which prevented me from speaking of the freedom which we have to follow good and evil was the fact that I wanted to avoid as far as possible all theological controversies and stay within the limits of natural philosophy. But I agree with you that whatever there is an occasion for sinning, there is indifference; and I do not think that in order to do wrong it is necessary to see clearly that what we are doing is evil. It is sufficient to see it confusedly, or merely to remember that we once judged it to be so, without in any way seeing it - that is, without paying attention to the reasons which prove it to be so. For if we say it clearly, it would be impossible for us to sin, as long as we saw it in that fashion; that is why they say that whoever sins does so in ignorance. ....; from which it follows, in my view, that this freedom does not consist in indifference." 115 [Hervorhebung hinzugefügt]

Mit dieser eher theologisch belasteten Erklärung erweist sich Descartes' Willenslehre erneut als unvollständig, was die Freiheit des Willens anbelangt. Solange Descartes dem Willen auch bei einem falschen Urteil keine Freiheit einräumt, sich selbst zu bestimmen, so bleibt seine Selbstbestimmungsfreiheit mit seinem Aufmerksamkeitsargument ein halber Nachweis. Es geht hier in der Sache um eine wichtige Frage, nämlich ob sich der Wille auch gegen das Gewissen frei entscheidet bzw. ob die Unfreiheit des Willens angenommen werden muss, wenn sich der Wille gegen das Gute entscheidet. Freilich ist das letztgenannte Gute eine Interpretationssache, die in der Macht seines Richters steht, der dann jede ihm unliebsame Entscheidung als unfrei oder einen verkehrten Gebrauch der Freiheit titulieren kann. Sich der Bedeutung dieser Fragen wohl bewusst setzt sich Descartes in seinem zweiten Brief v. 9. Februar 1645 erneut mit dem Problem auseinander, ob der Wille in den genannten Fällen doch eine Möglichkeit hat, sich anders als für das Gute zu entscheiden. So führt er an:

"As for freedom of the will, I entirely agree.... I would like you to notice that 'indifference' in this context seems to me strictly to mean that state of the will when it is not impelled one way rather than another by any perception of truth or goodness. This is the sense in which I took it when I said that the lowest degree of freedom is that by which we determine ourselves to things to which we are indifferent. But perhaps others mean by 'indifference' a positive faculty of determining oneself to one or other of two contraries, that is to say, to pursue or avoid, to affirm or deny. I do not deny that the will has this positive faculty. Indeed, I think it has it not only with respect to those actions to which it is not pushed by

114 Zum Vergleich der cartesianischen Willensfreiheit mit der scholastischen Sündenlehre siehe ausführlich Embry, Descartes on Free Will, PPR 2016, S. 8 ff.

115 CSMK, 234 (AT IV, 117 f.); zur Interpretation dieser Stelle siehe auch Gilbert, Pac. Philos. Q. 86 (2005), S. 211 f. 
any evident reasons on one side rather than on the other, but also with respect to all other actions; so that when a very evident reason moves us in one direction, although morally speaking we can hardly move in the contrary direction, absolutely speaking we can. For it is always open to us to hold back from pursuing a clearly known good, or from admitting a clearly perceived truth, provided we consider it a good thing to demonstrate the freedom of our will by so doing." 116 [Hervorhebung hinzugefügt]

In seinem zweiten Brief kommt Descartes bei näherem Hinsehen Mesland beachtlich entgegen. Er scheint bereit zu sein, dem Willen in allen Fällen die Freiheit zu gewähren, sich selbst zu bestimmen. Allerdings fügt er für den Fall, dass sich der Wille gegen eine klar erkannte Wahrheit entscheidet, eine weitere Bedingung hinzu: der Wille muss dabei einen anderen Zweck verfolgen, der ebenfalls gut ist. Wann so etwas der Fall sein kann, lässt Descartes offen. ${ }^{117}$ Außerdem bleibt unklar, was er an dieser Stelle mit der moralischen und absoluten Möglichkeit meint, zumal seine genannte Bedingung ja moralisch ist. Und Descartes weißt wohl, dass das philosophische Interesse nicht in moralischen Erklärungen liegt. Dies belegen seine weiteren Ausführungen in dem Brief, bei denen er die Willensfreiheit entsprechend einer Differenzierung erneut überprüft, die eigentlich von Anfang an seinem Willensverständnis zugrunde lag, nämlich die zeitliche Trennung des sich entschließenden Willens und der Fällung des Urteils selbst. ${ }^{118}$ So schreibt er:

"It must be noted also that freedom can be considered in the acts of the will either before they are elicited, or after they are elicited.

Considered with respect to time before they are elicited, it entails indifference in the second sense [self-determination] but not in the first [not impelled]. ...For a greater freedom consists either in greater facility in determining oneself or in a greater use of the positive power which we have of following the worse although we see the better. If we follow the course which appears to have the most reasons in its favour, we determine ourselves more easily,; but if we follow the opposite, we make more use of that positive power; ....

But freedom considered in the acts of the will at the moment when they are elicited does not entail any indifference taken in either the first or the second sense; for what

116 CSMK, 245 (AT IV, 173 und 174).

117 In der Tat ist es kaum vorstellbar, wann jemand berechtigterweise gegen ein klar erkanntes Gut verstoßen soll, sodass er allein dadurch die Existenz seiner Willensfreiheit gegen außen beweist. Sollte es nicht auf einen solchen Anwendungsbereich der gegen das Gute gerichteten Willensentscheidungen kommen, so kommen etwa Fälle in Betracht, in denen eine Person etwa gegen eine klar erkannte gute Pflicht verstößt, um eine Gefahr gegen sich selbst oder einen anderen abzuwenden. Hier könnte man annehmen, dass eine Demonstration der Freiheit des Willens, der ja immer auf das Gute gerichtet ist, eine gute Sache ist, weil er das Böse zum Schutze des immer das Gute begehrenden Willens abwendet. Dasselbe gilt den Fällen, in denen eine Person sich selbst oder einen anderen gegen Angriffe der Drittpersonen dadurch verteidigt, in dem sie gegen ein Gebot ausnahmsweise verstößt. In Anbetracht dieser Beispiele stellt sich die Frage, ob und inwiefern Descartes seine juristischen Kenntnisse in seine Philosophie des Geistes einfließen ließ. Aus Zeitgründen muss diese Frage an dieser Stelle leider unbeantwortet bleiben.

118 Für mehr dazu siehe oben II.2. 
is done cannot remain undone as long as it is being done. It consists simply in ease of operation; and at that point freedom, spontaneity and voluntariness are the same thing." 119 [Hervorhebung hinzugefügt]

Mit der Unterscheidung zwischen dem Willen, der noch nicht endgültig in eine Richtung „entlockt“ ist, und dem Moment der Entscheidung bringt Descartes seine Willenstheorie endlich auf einen Stand, in dem die Freiheit des Willens nicht nur final erklärt werden kann, sondern auch einer empirischen Betrachtung zugänglich ist. Descartes räumt ein, dass der Wille spätestens zur Zeit der Entscheidungsfällung, als absolute Spontanität, weder in Anbetracht der Gesetze des Guten und des Bösen noch aus der Sicht einer unbeschränkten Selbstbestimmungsfreiheit erklärbar ist: der Wille hat etwas getan, was hätte nicht ungetan bleiben können! Dabei bleibt dem Willen eine Selbstbestimmungsfreiheit nur in der Phase des „Entlocktwerdens“ bzw. des Sich-Entschließens, jedoch mit den oben genannten zwei Einschränkungen (Aufmerksamkeitsargument und die Entscheidung gegen Gewissen mit einem guten Grund). Die Willentlichkeit und Freiheit, die der Wille erfährt, wenn er ein klar erkanntes Gut vor sich hat, sind zwar für Descartes identisch mit dieser absoluten Spontanität. Sie definieren ihn jedoch noch, weil etwas getan ist, das hätte nicht ungetan bleiben können.

\section{Zwischenergebnisse}

Nach dem oben Gesagten lässt sich feststellen, dass Descartes bereits bei den Einwänden gegen die Meditationen Schwierigkeiten hinsichtlich seiner Erklärung der finalen Willensfreiheit bekommt. Auch wenn er in den Meditationen Hobbes' mit der Wiederholung seiner eigenen Annahmen abtun konnte, so hat er später in zwei Briefen an Mesland seine Willenslehre dahingehend modifiziert, dass der Wille eine gewisse Selbstbestimmungsfreiheit hat und seinen Charakter des auf das Gute gerichteten menschlichen Vermögens dabei weiterhin beibehält. Spätestens bei der Entscheidungsfällung ist dann der Wille aber pure Spontanität. Allerdings wird der cartesianische Wille mit der Erklärung, die Descartes als Beleg dafür einbringt, auch mysteriös: "for what is done cannot remain undone as long as it is being done". Es bleibt also unklar, ob sich der cartesianische Wille somit einer hypothetischen Prüfung entzieht, sodass er sich auch hätte anders verhalten können.

Bis jetzt wurde die Rolle Gottes beim Willen in Descartes' Theorie ausgeklammert. Zwar bringt diese für die hier oben gebliebene philosophische Frage keine Erklärung. Der cartesianische Wille würde aber unvollständig bleiben, wenn man auch Descartes' Ansichten nicht miteinbezieht, die die Verbindungen zwischen Gott, dem menschlichen Geist und seiner Willensfreiheit erklären. Darauf wird nun unten eingegangen.

\section{Gott und der menschliche Geist}

Wie bereits oben festgestellt, hat Descartes am Ende der Dritten Meditation lediglich seine eigene Existenz als Geist bzw. ein denkendes Ding und die Existenz Gottes bewiesen. Aus

119 CSMK, 246 (AT IV, 175.) 
diesen zwei Grundsätzen weitere Erkenntnisse hinsichtlich der physischen Welt mit demselben Beweismaß, das er für die beiden erreicht haben will, zu deduzieren ist unmöglich. Es liegt auf der Hand, dass Descartes an dieser Stelle Hilfe benötigt, die unter diesen Umständen nur von Gott kommen kann. ${ }^{120}$ Gleich zu Beginn der Vierten Meditation rollt Descartes seinen ontologischen Gottesbeweis erneut auf, den bereits er in der Dritten Meditation erbracht hatte, ${ }^{121}$ und fügt erstens folgendes hinzu:

"Und ich scheine bereits einen Wege zu gehen, auf dem man von dieser Kontemplation des wahren Gottes, in dem nämlich alle Schätze der Wissenschaften und der Weisheit verborgen sind, zur Erkenntnis der übrigen Dinge gelangen kann. “122 [Hervorhebung hinzugefügt]

Nun muss Descartes eine Sache feststellen: sein Geist irrt sich immer wieder. Könnte es nicht sein, dass Gott das veranlasst? Auf diese Dritte Szenario seines berühmten Zweifelsverfahrens ${ }^{123}$ gibt Descartes folgende Antwort:

"Erstens ...erkenne ich es als unmöglich dass Gott mich jemals täuscht. In aller Täuschung oder Betrug wird nämlich irgendeine Unvollkommenheit angetroffen, .... täuschen zu wollen [ist] Schlechtigkeit oder Schwäche, und demnach [kann] in Gott nicht stattfinden. ${ }^{124}$ [Hervorhebung hinzugefügt]

Der wahre Gott ist für Descartes vollkommen. Damit ist allerdings immer nicht noch alles getan, damit der Mensch selbst Empfänger verlässlicher und sicherer Erkenntnisse über körperliche Dinge sein kann. Denn der Geist ist weiterhin nur noch ein denkendes Ding und als solche kann er nur denken, und nichts mehr. Es reicht nicht, dass er den wahren Gott erkannt hat, der ihn nie täuschen würde. Auch seine kognitive Kapazität muss dergestalt sein, dass er mit dem genannten Beweismaß in der physischen Welt Fuß fassen kann. ${ }^{125}$ Diesbezüglich führt Descartes zweitens wie folgt aus:

"Außerdem erfahre ich, dass es in mir ein gewisses Urteilsvermögen gibt, das ich sicherlich von Gott erhalten habe, wie alles übrige auch, das in mir ist; und da er mich nicht täuschen will hat er es mir demnach als ein solches gegeben, dass ich mich niemals irren kann, solange ich es richtig verwende. "126 [Hervorhebung hinzugefügt]

$120 \mathrm{Zu}$ der zentralen Rolle des Gottes in der cartesianischen Erkenntnistheorie und auch Metaphysik siehe Perler, Descartes, S. 188.

121 AT VII, 45; zu Gottesbeweisen bei Descartes ausführlicher bei Perler, Descartes, S. $201 \mathrm{f}$.

122 AT VII, 52.

123 Hierzu im Allgemeinen siehe Jaspers, Descartes und die Philosophie, S. 6.

124 AT VII, 53.

125 Zur Aufhebung des Zweifels hinsichtlich kognitiven Grundlagen des Geistes bei Descartes siehe auch Perler, Descartes, S. 78.

126 AT VII, 53; ähnlich auch in AT VII, 58. 
Der Verstand und Wille, welche am Erkenntnisakt des Geistes beteiligt sind, ${ }^{127}$ haben also auch eine göttliche Natur. ${ }^{128}$ Descartes führt außerdem an, dass der Wille im Ergebnis göttliche Wahrheiten begehrt: ${ }^{129}$

".. Da er [der Mensch] bereits eine von Gott bestimmte Natur alles Guten und Wahren vorfindet und seinen Willen gar nicht auf irgendetwas anderes richten kann... "130 [Hervorhebung hinzugefügt]

Des Weiteren ist Descartes davon überzeugt, dass die göttliche Gnade neben dem natürlichen Licht des Verstandes die zweite Ursache der Wahrheit ist. Diesbezüglich führt er aus:

".., dass die Klarheit, bzw. Transparenz, durch die unser Wille zur Zustimmung bewegt werden kann, zweifach ist: nämlich durch das Natürliche Licht und durch die göttliche Gnade. ... dieser formale Grund besteht ... in einem bestimmten inneren Licht, durch das wir uns, wenn wir von Gott übernatürlich erleuchtet werden, darauf verlassen, dass das von ihm selbst offenbart ist, was uns zu glauben geboten wird, und dass er unmöglich lügen kann. Und das ist sicherer als alles Licht der Natur und aufgrund des Lichts der Gnade oft auch evidenter. "131 $[$ Hervorhebung hinzugefügt]

Was die Irrtümer des Geistes anbelangt, die er trotz der göttlichen Grundeinstellung des Willens begeht, wurden bereits oben ausführliche Erklärungen gemacht. ${ }^{132}$ Allerdings bleiben Descartes eingangs gestellte Behauptung und die gegebene Vision, dass "alle Schätze der Wissenschaften“ in Gott „verborgen sind“ und er mit dem von ihm gegebenen Urteilsvermögen „zur Erkenntnis der übrigen Dinge gelangen kann“, auch nach der Feststellung der Irrtumsanfälligkeit des Willens weiterhin ziemlich anspruchsvoll. Er fühlt sich wohl genötigt, über die metaphysischen Ursachen des sich immer wieder irrenden Willens mehr sagen zu müssen. Diesbezüglich führte er erstens auf:

".. Da der Wille nämlich nur in einem einzigen Ding, und gewissermaßen im Unteilbaren besteht, scheint seine Natur es nicht zu erlauben, dass irgendetwas von ihm weggenommen werden könnte. ".133 [Hervorhebung hinzugefügt]

Damit bringt Descartes ein Argument ein, das belegen soll, dass der Wille von einer solchen Beschaffenheit ist, die sogar nicht darin beschränkt werden kann, sich zu irren. ${ }^{134}$ Für die

127 Hierzu siehe oben II.1. und 2.

128 Hierzu siehe oben I.3.

129 Hierzu siehe oben II.2.

130 AT VII, 431.

131 AT VII, 147.

132 Hierzu siehe oben II.2.

133 AT VII, 60.

134 Zur Unbeschränktheit des Willens sagt Descartes außerdem:

„auf Alles, was Gegenstand eines anderen Willens oder des unermesslichen Willens in Gott sein kann, sich unser Wille erstrecken kann."(AT VIIIA, 18). 
Bescherung mit einem unteilbaren und unendlichen Willen muss der Mensch allerdings Gott danken, weil es gerade der Wille ist, mit dem der Geist die Erkenntnis der Existenz Gottes erlangt hat. So führt Descartes aus:

"..., erfahre ich doch, dass diese Freiheit, bzw. der Wille durch überhaupt keine Grenzen eingeschränkt wird... und zugleich bilde ich die Idee eines anderen, viel größeren, ja sogar eines größten und unendlichen, und erfasse allein dadurch, dass ich die Idee dieses Vermögen bilden kann, dass diese Idee der Natur Gottes zukommt. ... Es ist allein der Wille, bzw. die Freiheit der Willkür, die ich in mir als so groß erfahre, dass ich die Idee keines größeren Dinges auffassen kann - so dass es in erster Linie diese Idee ist, im Hinblick worauf ich mich gewissermaßen als Bild und Abbild Gottes verstehe. “135

Zweitens relativiert er seinen erwähnten Anspruch, was ebenfalls belegen soll, warum sein Geist „alle Schätze der Wissenschaften“ doch nicht in Erfahrung bringen kann. So sagt Descartes, dass der Mensch als Subjekt des Erkennens doch ein schwaches Wesen ist und ihm die Mängel eines Geschöpften innewohnen. Dazu äußert er sich an zwei Stellen in der Vierten Meditation:

"Weil das von ihm erhaltene Vermögen, das Wahre zu beurteilen, in mir nicht unendlich ist, passiert es, dass ich mich irre. "136 [Hervorhebung hinzugefügt]

"es liegt in der Beschaffenheit des endlichen Verstandes, dass er vieles nicht einsieht, und in der Beschaffenheit des geschaffenen Verstandes, dass er endlich ist.. "137 [Hervorhebung hinzugefügt]

Bis jetzt hat Descartes versucht zu erklären, warum der menschliche Geist immer wieder Irrtümer begeht, obwohl er mit dem ihm von Gott gegebenen Urteils- und Erkenntnisvermögen ausgestattet ist. Nun stellt sich die Frage, wie und warum Gott überhaupt Irrtümer erlaubt oder duldet, die ja erkenntnistheoretisch für die Menschen nicht nur Dunkelheit bedeuten, sondern auch moralisch verheerende Folgen haben. ${ }^{138}$ Diesbezüglich äußert sich Descartes in der Vierten Meditation an zwei Stellen wie folgt:

"Und so sehe ich sicher ein, dass Irrtum, insofern er ein Irrtum ist, kein reales Etwas ist, das von Gott abhinge, sondern nur ein Mangel“139 [Hervorhebung hinzugefügt]

"... Die Privation aber, in der allein der formale Grund der Falschheit und der Verantwortung besteht, bedarf keiner Unterstützung Gottes, weil sie kein Ding ist, und deshalb darf man sie auf Gott als Ursache bezogen auch nicht als Privation, sondern lediglich als Negation bezeichnen. ${ }^{\text {"140 }}$ [Hervorhebung hinzugefügt]

135 AT VII, 57; so auch bereits in seinem Brief v. 25. Dezember 1639 an Mersenne (AT II, 628).

136 AT VII, 54.

137 AT VII, 60.

$138 \mathrm{Zu}$ dieser Fragestellung siehe etwa AT VIIIA, 19.

139 AT VII, 54.

140 AT VII, 60; so auch in AT VIIIA, 17. 
Aus diesen Stellen ist es zu entnehmen, dass die Irrtümer des Geistes laut Descartes keine Realität in dem Reich des wahren Gottes haben, das nur aus Wahrheiten besteht. Dass sie dennoch dem Geist immer wieder unterlaufen, gewährt ihnen keine Existenz, die wahr sein muss und stets von Gott abhängt. ${ }^{141}$

Schließlich stellt sich die Frage nach dem metaphysischen Sinn der Stellung des Menschen, der einen begrenzten Verstand hat, sich deswegen in einer unvermeidbaren oder vermeidbaren Weise immer wieder irrt und eines unklaren Urteils nicht immer enthalten kann. Gott ist nämlich in Descartes Konzeption des Universums so allmächtig, dass er den Menschen auch ohne diese Mängel hätte erschaffen können. Zur Rechtfertigung des Status quo, den Descartes in der Vierten Meditation beschreibt, bringt er drei Argumente ein.

Erstens ist es nach Descartes selbstverständlich, dass der Mensch als Geschöpft eines vollkommenen Schöpfers keinen unbegrenzten Verstand hat, weil er sonst vielleicht gottesgleich wäre:

"Es aber nicht besonders verwunderlich ist, dass ich mich täusche, .....: insofern ich nicht selbst ein höchstes Seiendes bin, und mir außerordentlich viel mangelt “142 [Hervorhebung hinzugefügt]

Zweitens sieht Descartes nicht ein, dass der Mensch von seinem Schöpfer etwas verlangen kann:

".Kein Recht aber habe ich, mich zu beklagen, dass Gott mir in der Welt eine Rolle zuzuweisen gewillt war, die weder die vorzüglichste noch die vollkommenste von allen ist. ${ }^{\text {"143 }}$ [Hervorhebung hinzugefügt]

Insgesamt findet es Descartes übermütig, dass der Mensch, auch „die Zwecke Gottes“ untersuchen können will. ${ }^{144}$ Und drittens hofft Descartes das Vorhandensein eines transzendentalen Sinns in der Gesamtordnung der Dinge einschließlich der menschlichen Existenz:

"...; aber deswegen kann ich doch nicht bestreiten, dass in gewisser Hinsicht dadurch eine größere Vollkommenheit in der Gesamtheit der Dinge insgesamt liegt, weil bestimmte Teile von ihr gegen Irrtümer nicht gefeit sind, andere aber sehr wohl, als wenn alle völlig gleich wären. ${ }^{1145}$ [Hervorhebung hinzugefügt]

Soweit sich Descartes hierbei auf theologische Anschauungen bezieht, betrifft dies die philosophische Willensfrage nicht in dem Maße, als wenn es um die Rolle Gottes geht.

141 Zur Idee des Gottes als „höchste Wahrhaftigkeit“ siehe auch AT VIIIA, 16.

142 AT VII, 54; ähnlich auch in Prinzipien der Philosophie AT VIIIA, 18.

143 AT VII, 60; ähnlich auch in AT VII, 57; AT VIIIA, 19.

144 AT VII, 55; ähnlich auch in seinem Brief v. 2 Mai 1644 an Mesland, CSMK, 235 (AT IV, 119); hierzu siehe auch Jaspers, Descartes und die Philosophie, S. 69.

145 AT VII, 60. 
Descartes' Willenslehre knüpft allerdings auch die weltliche Anwendung des Willensvermögens daran, dass Gott darin involviert ist. Es stellt sich nun die Frage, ob und inwiefern dies mit der Willensfreiheit vereinbar ist. ${ }^{146}$

\title{
V. Gott und Freiheit des Willens
}

Wie bereits oben erwähnt, begehrt der Wille nicht nur das von Gott im Voraus bestimmte Gute und das Wahre, sondern er wird auch neben dem natürlichen Licht noch durch die göttliche Gnade zur Annahme des Guten bewegt. So schreibt Descartes in den Mediationen:

\begin{abstract}
"Es muss außerdem darauf hingewiesen werden, dass die Klarheit, bzw. Transparenz, durch die unser Wille zur Zustimmung bewegt werden kann, zweifach ist: nämlich durch das Natürliche Licht und durch die göttliche Gnade. "147 [Hervorhebung hinzugefügt]
\end{abstract}

Auf die Frage, ob dies die Freiheit des Willens vermindert, gibt Descartes eine verneinende Antwort. ${ }^{148}$ Denn der Wille besitzt begrifflich eine Natur, die nach Vollkommenheit sucht. Wenn Gott ihm dabei hilft und sozusagen den Weg abkürzt, wie kann man von einer Verminderung der Freiheit sprechen, die ja in der Erfüllung göttlicher Zwecke besteht? Außerdem fügt Descartes hinzu, dass die Gnade den menschlichen Willen nicht gänzlich bestimmt. ${ }^{149}$ Denn,

"dieses Licht richte[t] das Innerste unseres Denkens auf das Wollen aus und verminder[t] gleichwohl nicht die Freiheit. " ${ }^{150}$ [Hervorhebung hinzugefügt]

Descartes argumentiert weiter, dass der Wille auch in der Tat der Gnade nicht immer folgt. Diesbezüglich gibt er aus dem Bereich des Glaubens das folgende Beispiel:

"Wenn Türken und andere Ungläubige die christlichen Religion nicht annehmen, gehen sie nicht dadurch fehl, dass sie dunklen Dingen nicht zustimmen wollen, da diese nun einmal dunkel sind, sondern entweder dadurch, dass sie der sie innerlich bewegende göttlichen Gnade Widerstand leisten, oder dadurch, dass sie sich der Gnade unwürdig machen, indem sie in anderen Dingen fehlgehen. "151 $[$ Hervorhebung hinzugefügt]

Die Frage der göttlichen Gnade, insbesondere in ihrer letzten Einschränkung leitet einen gleich in einen Bereich über, in dem der menschliche Wille, seine Freiheit und Gott unter ganz anderen Aspekten untersucht werden können, die nicht unbedingt eine Philosophie des Geistes betreffen. So stellt sich die Frage, wie der menschliche Wille überhaupt gegen die Gnade des allmächtigen Gottes Widerstand leisten kann etc.

146 Hierzu eingehend Gilbert, Pac. Philos. Q. 86 (2005), S. 210 ff.

147 AT VII, 147.

148 AT VII, 148

149 Siehe auch Gilbert, Pac. Philos. Q. 86 (2005), S. 219.

150 AT VII, 148.

151 AT VII, 148. 
Descartes' Willenslehre gerät an dieser Stelle in einen fatalistischen Determinismus, den Descartes aber unbedingt vermeiden möchte, weil die Freiheit des Willens dann kaum mehr Sinn hätte. ${ }^{152}$ So betont Descartes einerseits:
"But philosophy by itself is able to discover that the slightest thought could not enter into a person's mind without God's willing, and having willed from all eternity, that it is should so enter. ... God is the universal cause of everything in such a way as to be also the total cause of everything; and so nothing can happen without his will." 153 [Hervorhebung hinzugefügt]

Anderseits versucht Descartes seine Leser mit folgenden Warnungen und Tröstungen zu beschwichtigen:

"Hier können wir leicht uns in große Schwierigkeiten verwickeln, wenn wir versuchen, diese Vorherbestimmung Gottes mit der Freiheit unserer Willkür zu vereinigen und beide zugleich $z u$ begreifen. " ${ }^{154}$ [Hervorhebung hinzugefügt]

"Gleichwohl: Dass willentlich und frei dasselbe ist, erfährt jeder, der nur sich selbst betrachtet." 155

Schließlich gibt Descartes dem Willen und dessen Freiheit eine weltliche eher normative Rolle, die ihn vielleicht vor einem Vorwurf der Sinnlosigkeit retten kann. So schreibt er erstens in den Prinzipien der Philosophie:

"...und es bildet die höchste Vollkommenheit im Menschen, dass er durch seinen Willen, d.h. frei handelt. Damit ist er gewissermaßen der Urheber seiner Handlungen und kann deshalb gelobt werden. Denn die Automaten lobt man nicht wegen der genauen Ausführung aller Bewegungen, auf die sie eingerichtet sind, aber man lobt ihren Werkmeister wegen der genauen Verfertigung derselben, weil er dies nicht notwendig, sondern freiwillig vollführt hat. Aus demselben Grunde ist es mehr unsere Tat, dass wir das Wahre erfassen, wenn wir es erfassen, weil wir es mit Willen tun, als wenn wir es erfassen müssten. “156 [Hervorhebung hinzugefügt]

Der cartesianische Wille ist also für die weltliche Ordnung von Bedeutung, weil er den Anknüpfungspunkt dafür darstellt, dass Menschen ihre Handlungen objektiv zugerechnet werden. ${ }^{157}$ Jeder ist also Urheber seiner willentlichen Handlung. ${ }^{158}$ Zweitens führt Descartes in

152 Hierzu siehe auch Wee, Descartes and Leibniz, CJPh 36/3-2006, 387.

153 Brief v. 6. Oktober 1645 an Prinzessin Elisabeth CSMK, 272 (AT IV, 314); zur Interpretation der cartesianischen Grundannahme, dass Gott Ursache alles Existierenden ist, siehe Perler, Descartes, S. 108 f.

154 AT VIIIA, 20.

155 AT VII, 191; so auch in AT VIIIA, 20.

156 AT VIIIA, 19.

157 Hierzu siehe auch Fogal, Descartes, S. 21 f.

158 Für die Bedeutung des Prinzips der Urheberschaft in der modernen Debatte der Willensfreiheit siehe etwa Beckermann, s.1. 
den Leidenschaften der Seele an, dass dem Willen weitere Funktionen zukommen:

"... I see only one thing in us which could give us good reason for esteeming ourselves, namely, the exercise of our free will and the control we have over our volitions. For we can reasonably be praised or blamed only for actions that depend upon this free will. It renders us in a certain way like God by making us masters of ourselves, provided we do not lose the rights it gives us through timidity." 159

Der Wille liegt also der Selbstachtung zugrunde, weil sein Agent ihn wahrnimmt und sich über seine Kontrolle über seine Willentlichkeiten sowie Freiheit bei der Ausübung der Urteilskraft bewusst ist. Er gibt also seinem Agenten, die Zuversicht, sein eigener Herr zu sein. ${ }^{160}$ Und die Freiheit des Willens ist für Descartes das Prinzip des gesellschaftlichen Lob-TadelMechanismus. ${ }^{161}$

\section{Endergebnisse}

Wie die oben vorgenommenen Ausführungen zeigen, übernimmt der Wille in der cartesianischen Philosophie mehrere Rollen. Für den menschlichen Geist ist er zentrales Element des Bewusstseins, wenn er außer sich selbst etwas denkt. Ohne eine Beteiligung des Willens ist die kognitive Betätigung des Verstandes weder möglich noch bedeutsam. Der cartesianische Wille aktiviert den Verstand und bearbeitet die von ihm gelieferten Ideen zu einem Urteil. Er ist sowohl Begehren als auch Entschieden. Spätestens bei diesen zwei Eigenschaften des Willens hat Descartes zu erklären, was deren Prinzipien sind. Im Augenmerk dieser Debatte ist die Freiheit des menschlichen Willens. Eine rein philosophische Erklärung kann Descartes nicht liefern, weil seiner gesamten Erkenntnistheorie die Idee eines theistischen christlichen Gottes zugrunde liegt.

In der Tat ist der Wille nicht nur essentiell dafür, dass der menschliche Geist in einer rationalen Weise Erkenntnisse über die körperliche Welt gewinnt. Er ist auch die Schaltstelle, was die Verbindung zwischen dem menschlichen Geist und Gott anbelangt. Er ist allem voran ein Geschöpf Gottes und trägt seine Handschrift. Gott hat ihn nach Descartes so eingestellt, dass er das göttlich Gute, das ewig und absolut Wahre begehrt. Dies sind Annahmen, die mit einer rein metaphysischen Freiheit des Willens unvereinbar sind. In den Meditationen kann Descartes nur mit einem finalen Freiheitsverständnis erklären, dass der Wille einer durch den Verstand klar und deutlich erkannten Idee willentlich und spontan, also frei folgt. Im umgekehrten Fall, wo der Wille, der ja intrinsisch das Gute begehrt, eine solche Idee nicht vor sich hat, ist er keineswegs frei, wenn er die ihm vom Verstand gekennzeichneten Grenzen verkennend doch ein Urteil fällt und sich dabei irrt. Er ist hier nur indifferent, soweit es um seine Urteilskraft geht.

Mit diesen beiden Erklärungen stößt Descartes auf Kritik. Denn in beiden Fällen kann man nicht von einer wirklichen Freiheit des Willens reden. Attraktiver ist die Vorstellung, nachweisen

159 CSM I, 384.

160 Für die Bedeutung des Kontrollprinzips in der modernen Debatte der Willensfreiheit siehe etwa Beckermann, s.1.

161 Hierzu siehe Fogal, Descartes, S. 4; Jayasekera, Ergo 3/2016, S. 330. 
zu können, dass sich der Wille in allen Fällen selbst bestimmt. Später unternimmt Descartes zwei wichtige Versuche, seine Willenslehre entsprechend dieser Vorstellung zu ergänzen. Für den Fall, dass der Wille einer durch den Verstand klar und deutlich erkannten Idee spontan folgt, muss er einen Beweis erbringen, dass dies nicht mit Notwendigkeit geschieht und dass der Wille auch ein entgegengesetztes Urteil fällen kann. Dies gelingt ihm mit dem Argument der Aufmerksamkeit. Descartes hält für möglich, dass sich der Wille für einen Moment nicht mehr auf die Überzeugungskraft einer klar erkannten Wahrheit konzentrieren kann und eine andere Entscheidung fällt. Für den Fall, dass der Wille keine klar erkannte Wahrheit hat, tut sich Descartes schwer, eine Selbstbestimmungsfreiheit des Willens anzuerkennen. Sein Beispiel, bei dem er auch in so einem Fall die Freiheit des Willens zulassen würde, ist kaum verständlich: wie soll der Wille gegen eine klar erkannte Wahrheit entscheiden und dabei nur frei sein, wenn gerade eine solche Entscheidung die Freiheit des Willens demonstriert? Des Weiteren bemüht sich Descartes, eine Art der Selbstbestimmungsfreiheit des Willens in seine Lehre dadurch zu inkorporieren, dass er auf die Unbestimmbarkeit des Willens zwischen dem Sich-Entschließen und der Fällung des Urteils selbst hinweist. Im Ergebnis bleibt allerdings unklar, was Descartes damit genau meint. Für Verwirrung sorgt auch der Satz, den er in diesem Zusammenhang als Beleg anführt: for what is done cannot remain undone as long as it is being done.

Die philosophischen Rahmen verlässt Descartes schließlich, wenn er bei der konkreten Anwendung des Urteilsvermögens der göttlichen Gnade sowie der Prädetermination eine Rolle zukommen lässt. In dieser Phase einer Willenslehre kann Descartes nur mit Glaubensätzen seine Leser von der Richtigkeit seiner Ansichten überzeugen. Bemerkenswert ist allerdings, dass Descartes selbst innerhalb einer göttlich beherrschten Weltordnung dem Willen neben einer Selbstbestimmungsfreiheit - jedenfalls einer gefühlten - auch drei weitere Funktionen zuschreibt: die Willensfreiheit ermöglicht, dass äußerlich sichtbar gewordenen Handlungen Menschen als deren Urheber zugerechnet werden können. Die Willensfreiheit ist außerdem die Berechtigung eines gesellschaftlichen Lob-Tadel-Mechanismus. Und schließlich bedeutet die Willensfreiheit die Selbstkontrolle und erzeugt bei ihrem Agenten Selbstachtung.

Peer-review: Externally peer-reviewed.

Conflict of Interest: The author has no conflict of interest to declare.

Grant Support: The author declared that this study has received no financial support. 


\section{Bibliographie / References}

\section{Quellen}

Descartes, René, Regulae ad directionem ingenii (Regeln zur Ausrichtung der Erkenntniskraft). Übersetzt von Springmeyer, Heinrich/Gäbe, Lüder/Zekl, Hans Günter. Hamburg 1979.

Descartes, René, Discours de la méthode pour bien conduire sa raison et chercher la vérité dans les sciences (Abhandlung über die Methode, richtig zu denken und die Wahrheit in den Wissenschaften zu suchen). Übersetzt von Julius Heinrich von Kirchmann. Berlin 1870.

Descartes, René, Meditationes de prima philosophia in qua Dei existentia et animae immortalitas demonstratur (Die Meditationen über die erste Philosophie, in denen die Existenz Gottes und die Unterscheidung zwischen der menschlichen Seele dem Körper beweisen wird). Mit sämtlichen Einwänden und Erwiderungen. Übersetzt von Wohlers Christian. Hamburg 2009.

Descartes, René, Principia philosophiae, Amsterdam 1644. Übersetzung durch Julius Heinrich von Kirchmann von 1870.

Descartes, René, the Correspondence in: The Philosophical Writings of Descartes Volume III. Translated by John, Cottingham/Robert Stoothoff/Dugald, Murdoch/Anthony, Kenny. Cambridge 1991.

Descartes, René, Description of Human Body in: The Philosophical Writings of Descartes Volume I. Translated by John, Cottingham/Robert Stoothoff/Dugald, Murdoch. Cambridge 1985.

Descartes, René, the Passions of the Soul in: The Philosophical Writings of Descartes Volume I. Translated by John, Cottingham/Robert Stoothoff/Dugald, Murdoch. Cambridge 1985.

\section{Literatur}

Beckermann, Ansgar, Willensfreiheit - ein Überblick aus kompatibilistischer Sicht, 1-13. Abrufbar unter https://pub.uni-bielefeld.de/publication/2306223 [Letzter Abruf: 03.12.2017].

Embry, Brian, Descartes on Free Will and Moral Possibility. Philosophy and Phenomenological Research 2016, 1-9.

Fogal, Daniel, Descartes and the Possibility of Enlightened Freedom, 1-32. Abrufbar unter http://www. academia.edu/31312267/Descartes_and_the_Possibility_of_Enlightened_Freedom [Letzter Abruf: 04.12.2017].

Gilbert, Christopher, Grades of Freedom: Augustine and Descartes. Pacific Philosophical Quarterly 86 (2005), 201-224.

Haag, Johannes, Descartes über Willen und Willensfreiheit. Zeitschrift für philosophische Forschung. 60/42006, 483-503.

Jaspers, Karl, Descartes und die Philosophie. Dritte, unveränderte Auflage. Berlin 1956.

Jayasekera, Marie, Responsibility in Descartes's Theory of Judgement. Ergo, an Open Access Journal of Philosophy 3/2016, 321-347.

Kahl, Wilhelm, Die Lehre vom Primat des Willens bei Augustinus, Duns Scotus und Descartes. Straßburg 1886.

Perler, Dominik, René Descartes. 2., erweiterte Auflage. München 2006.

Rengier, Rudolf, Strafrecht. Allgemeiner Teil. 5. Auflage. München 2013.

Schulte, Peter, Willensfreiheit und Aufmerksamkeit bei Descartes. Zeitschrift für philosophische Forschung. 71/1-2017, 5-36.

Wee, Cecilia, Descartes and Leibniz on Human Free-Will and the Ability to Do Otherwiese. Canadian Journal of Philosophy. Volume 36/3-2006, 387-414. 
